

\title{
Impact of hydro-chemical conditions on structural and hydro-mechanical properties of chalk samples during dissolution experiments
}

\author{
M. Leger, D. Roubinet, M. Jamet, L. Luquot
}

\section{- To cite this version:}

M. Leger, D. Roubinet, M. Jamet, L. Luquot. Impact of hydro-chemical conditions on structural and hydro-mechanical properties of chalk samples during dissolution experiments. Chemical Geology, 2022, 594, pp.120763. 10.1016/j.chemgeo.2022.120763 . hal-03584946

\author{
HAL Id: hal-03584946 \\ https://hal.science/hal-03584946
}

Submitted on 22 Feb 2022

HAL is a multi-disciplinary open access archive for the deposit and dissemination of scientific research documents, whether they are published or not. The documents may come from teaching and research institutions in France or abroad, or from public or private research centers.
L'archive ouverte pluridisciplinaire HAL, est destinée au dépôt et à la diffusion de documents scientifiques de niveau recherche, publiés ou non, émanant des établissements d'enseignement et de recherche français ou étrangers, des laboratoires publics ou privés. 


\title{
Impact of hydro-chemical conditions on structural and hydro-mechanical properties of chalk samples during dissolution experiments
}

\author{
M. Leger ${ }^{\mathrm{a}, *}$, D. Roubinet ${ }^{\mathrm{a}}$, M. Jamet ${ }^{\mathrm{a}}$, L. $_{\text {Luquot }}{ }^{\mathrm{a}}$ \\ ${ }^{a}$ CNRS, Geosciences Montpellier, Université de Montpellier, Montpellier, France
}

\section{Highlights}

2

3

4

5

6

7

9 


\section{Abstract}

The importance of karst reservoirs for water resources, and their complexity in terms of structural properties and hydraulic responses, require a better understanding of the formation and location of conduits in these systems, in particular for chalk reservoirs. For this purpose, we conduct laboratory experiments of acid solutions injection in chalk core samples using a homemade experimental device. Three different flow rates and two acid concentrations are applied on six samples at atmospheric conditions with Peclet numbers ranging from 0.1 to 1.7 and Damköhler numbers from $10^{-6}$ to $10^{-3}$. Hydraulic and chemical measurements are carried out during the experiments, while petrophysical characterization is conducted on the samples before and after the reactive percolation experiments. The analysis of these data shows an increase in permeability of the rock samples due to calcite dissolution, which is associated with the creation of preferential conduits. We also observe that the initial structural properties control the dissolution regime with the following main conclusions. For homogeneous systems, the overall dissolution rate and percolation time are directly linked to the considered flow rate and injected acid concentration. For heterogeneous systems, (i) pre-existing large-scale structural heterogeneities control the location of newly created paths while reducing the value of the dissolution rate, and (ii) micro-scale heterogeneities result in highly heterogeneous general structures, which are responsible for channels formation while applying low Damköhler numbers. 


\section{Keywords}

Chalk, Reactive transport, Dissolution regime, Micro-tomography, Karst, Wormholes

\section{Introduction}

Carbonate rocks are widely studied because of their important presence on the Earth's surface and their use for human activities, including geothermal energy and resources extration (Rege and Fogler, 1989; Chilingarian et al., 1992; Noiriel et al., 2005; Moore and Wade, 2013). These formations, which present scale-dependent complex properties (Choquette and Pray, 1970; Lucia, 1983; Lønøy, 2006), are characterized by a strong reactivity. This is illustrated by the dissolution of carbonate rocks, which is the result of thermodynamical disequilibrium between fluid and rock (Ford and Williams, 2007) and leads to the formation of karsts.

Numerous laboratory experiments and numerical simulations have been conducted on various carbonate rocks and under variable conditions in order to understand how the dissolution process occurs in such systems. Golfier et al. (2002) linked the initial conditions of dissolution and the resulting dissolution patterns (from uniform dissolution to the formation of compact wormholes) with the use of the adimensional Péclet (Pe) and Damköhler (Da) numbers (Lasaga, 1984; de Marsily, 1986; Daccord et al., 1993). This is in the line with numerous laboratory and theoretical experiments that demonstrate the determinant impact of the fluid rate and structural properties on the developed dissolution regimes (Fredd and Fogler, 1998; Singurindy and Berkowitz, 2003; Noiriel et al., 2004; Luquot and Gouze, 2009; Smith et al., 
2013; Vialle et al., 2014; Menke et al., 2015; Lebedev et al., 2017). Nevertheless, the Damköhler number is difficult to calculate as demonstrated by the large number of techniques that have been considered for this purpose (Lasaga, 1984; Knapp, 1989; Luquot and Gouze, 2009; Garcia-Rios et al., 2015; Menke et al., 2015; Noiriel and Daval, 2017), mostly because of the complexity of determining the reaction rate $K\left[s^{-1}\right]$. As a result, linking the dissolution rate and regime, as well as the reaction localization, to the $\mathrm{Pe}$ and Da numbers is still challenging.

The structure is also known to be an important parameter in carbonate rock dissolution. Pore size distribution can be heterogeneous in carbonate rocks and affect the flow path through the rock. These heterogeneities in the pore structure lead to different dissolution mechanisms that occur inside the rock sample (Hoefner and Fogler, 1988; Noiriel et al., 2005; Vialle et al., 2013). For example, in fractured rock samples, the dissolution usually occurs along the main heterogeneities favouring a transport-controlled mechanism (Elkhoury et al., 2013; Garcia-Rios et al., 2017). In order to study how and where the dissolution occurs, as well as the resulting changes on the rock sample properties, various laboratory dissolution experiments were conducted on carbonate rocks at room and reservoir conditions on limestone rocks (Fredd and Fogler, 1998; Noiriel et al., 2004, 2005; Luquot and Gouze, 2009; Noiriel et al., 2009; Gharbi et al., 2013; Mangane et al., 2013; Vialle et al., 2013; Luquot et al., 2014a; Molins et al., 2014; Vialle et al., 2014; Steefel et al., 2015; Menke et al., 2017; Lebedev et al., 2017) and dolomite at reservoir conditions (Smith et al., 2013; Tutolo et al., 2014; Luhmann et al., 2014). Although most of these studies try to express the dissolution features with, 
again, the Pe and Da numbers, they finally defined new dissolution features linked to the rock heterogeneities (Noiriel et al., 2009; Luquot et al., 2014a; Menke et al., 2017).

Most of the previous studies have been performed in (almost) homogeneous limestone rocks that are not representative of natural complex rocks such as chalk. The latter is characterized by highly heterogeneous structures composed of variable-size shells and a high proportion of very small pores (Fabricius, 2007). This special structure makes the determination of a characteristic length difficult, inducing complications in the adimensional numbers definition. It also results in unstable structures that are difficult to reproduce experimentally and thus not well studied. A recent study has investigated the different dissolution patterns occurring inside chalk by injecting $\mathrm{CO}_{2}$ at elevated pressure using in situ X-ray imaging (Yang et al., 2018). They demonstrate the high sensibility of chalk to reactive fluid compared to coupled geochemical and geomechanical processes, and show that the microstructure is highly affected by material compaction, fracturing and grain relocation. Nevertheless, they did not perform these experiments under different dissolution regimes and the hydraulic properties are not monitored.

The present work attempts to give some answers on how conduits in chalk form and what are the properties responsible for it. To this end, acid injections are conducted on six core samples of chalk at atmospheric conditions. Three different flow rates and two acid concentrations are applied to the samples with a homemade experimental device. The chemical and hydrodynamic properties are recorded during the experiments, and the samples are characterized with laboratory and images methods before and after experi- 
ments. Evolution of all these properties coupled with the initial conditions, is analyzed in order to observe in which proportion they are responsible for the changes induced by the acid flowing through the rock.

\section{Materials and Methods}

\subsection{Rock properties and characterization}

We study six chalk samples, named N02, N03, N04, N05, N08 and N09, that were cored in a unique flint chalk block from the lower Senonian located in north-western France. The length of the cores is 20-30 mm and their initial diameter of $18 \mathrm{~mm}$ results in $25 \mathrm{~mm}$ when surrounded with epoxy resin and PVC pipe. Although most of the samples present pores in stick shape without preferential orientation and with pre-existing fractures, some discrepancies are observed between the samples (Figure 1).

Petrophysical non-destructive measurements that are carried out on the cored samples are described in Leger and Luquot (2021) under the rock named Normandie (label N). It includes rock conductivity monitoring, structural properties computation (formation factor $F$, cementation index $m$ and electrical tortuosity $\tau_{e}$ ), pore size characterization with centrifugation, X-ray tomography calculations resulting in the porosity of the macro phase, permeability, hydraulic tortuosity $\tau_{h}$, relative pores size $\frac{S}{V}$, proportion of the percolating phase $P V$ and the pore size distribution represented as chord length probability distribution. In addition to this data, 3D images obtained from X-ray tomography calculations are used to extract the pore network of the samples and their corresponding permeability (see details in Electronic Supplement). 


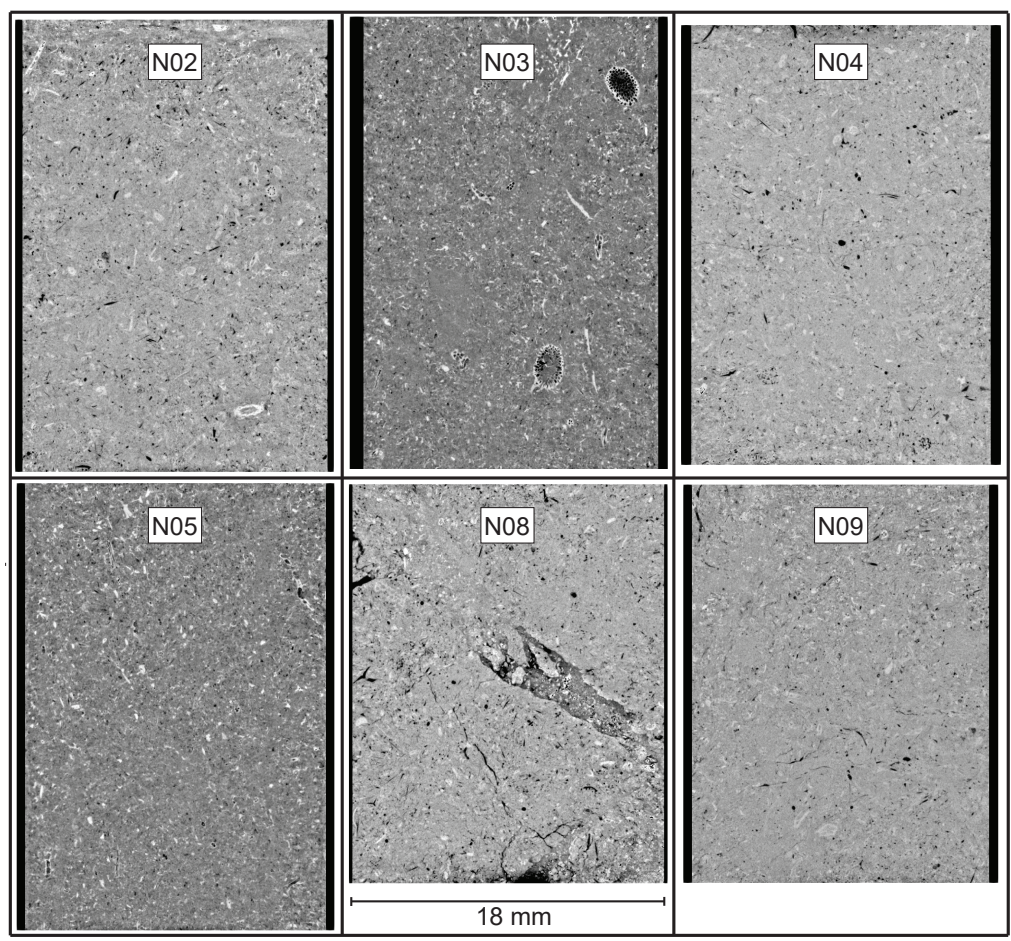

Figure 1: 2D vertical slices from 3D X-ray micro-tomography (XRMT) images of the initial structure of the six studied samples. N05 is the most homogeneous sample while N08 and N09 present a pre-existing filled channel and some pre-existing micro-cracks, respectively.

\subsection{Experimental protocol and output}

An acidic fluid composed of acetic acid $\mathrm{CH}_{3} \mathrm{COOH}$ and sodium acetate $\mathrm{CH}_{3} \mathrm{COONa}$, which are diluted in water previously equilibrated with chalk, is injected through the core samples at atmospheric pressure and temperature using a homemade experimental device (Figure 2). The airtight sample holder allows an injection distributed over the sample surface. The $\mathrm{pH}$ and conductivity of the injected solution, as well as the difference in pressure between the sample inlet and outlet, are continuously monitored, while $\mathrm{pH}$, conductivity and cation concentrations of the outlet fluid are measured with 
regular sampling of $5 \mathrm{~mL}$ using the ICP (Ion Concentration Polarization) method for ion concentration. The protocol that is applied to each sample is described in details in Appendix A.1.

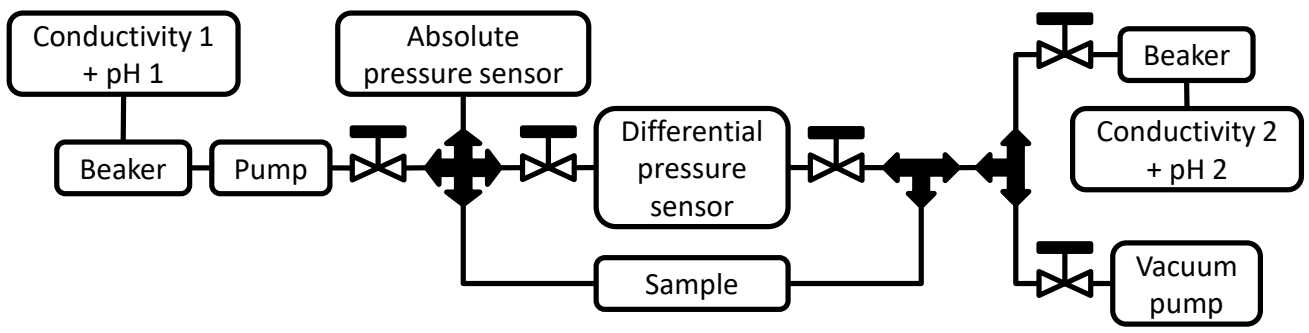

Figure 2: Experimental homemade percolation device composed of a beaker with JUMO sensors, a peristaltic pump (Gilson, MiniPuls3), an absolute pressure (Gems) and a differential pressure (Keller) sensor, Swagelok connectors, and a continuous acquisition system Labjack.

Five different experimental conditions are considered with two acidic concentrations $\left(C_{0}^{1}\right.$ and $\left.C_{0}^{2}\right)$ and three flow rates $\left(P e_{1}, P e_{2}\right.$ and $\left.P e_{3}\right)$ defined by the Peclet number $P e=\frac{u l}{d}$, with $u$ the average flow velocity injected in each sample $[\mathrm{m} / \mathrm{s}], l$ the characteristic length of the pores $[\mathrm{m}]$ and $d$ the molecular diffusion in calcite $\left[\mathrm{m}^{2} / \mathrm{s}\right]$ set to $5 \times 10^{-9} \mathrm{~m}^{2} / \mathrm{s} . C_{0}^{1}$ and $C_{0}^{2}$ are set to $10^{-3}$ and $10^{-2} \mathrm{~mol} / \mathrm{L}$ of acid concentration, respectively, and the ranges of $P e_{1}$, $\mathrm{Pe}_{2}$ and $\mathrm{Pe}_{3}$ are $[0.01,0.2],[0.05,1.5]$ and [0.1,3.25], respectively. The latter values come from the pore size distributions provided in Leger and Luquot (2021) that are used here to define the characteristic length $l$, as done in previous studies (Vialle et al., 2014; Menke et al., 2016). Table 1 summarizes the experimental conditions that are applied to the samples with N03 and N08 being reproduction experiments. For each experiment, the evolution of the sample porosity $\phi(t)$ along time is deduced from the measured concentration of Ca ions with the methodology described in Appendix A.2. 


\begin{tabular}{c|cc}
$P e$ & $C_{0}^{1}$ & $C_{0}^{2}$ \\
\hline$P e_{1}$ & N04 & N09 \\
$P e_{2}$ & N02 & N03 \& N08 \\
$P e_{3}$ & & N05
\end{tabular}

Table 1: Experimental conditions of the experiments. $P e_{1}, P e_{2}$ and $P e_{3}$ are associated with the flow rates $2.9,25.2$ and $63 \mathrm{~mL} / \mathrm{h}$, respectively.

After dissolution, the sample is submitted to the same characterization methods as before the experiments (Section 2.1) in order to evaluate the impact of the percolation experiment on the rock properties. This includes pore size characterization by centrifugation in the direction of the pressure difference and in the opposite one, which allows to characterize the possible anisotropy.

\section{Results}

\subsection{Hydro-chemical results from percolation experiments}

Figure 3 displays the evolution over time of the outlet $\mathrm{pH}$ values, fluid conductivities $\left(\sigma_{f}\right)$ and calcium concentration $[\mathrm{Ca}]$ recorded during the percolation experiments. We observe that all these values are higher than the inlet ones, indicating that the main chemical reaction occurring during the experiments is calcite dissolution. We also observe that the shapes of these curves are similar, with a plateau at the beginning of the experiment that is followed by a decrease. At low inlet acid concentration $C_{0}^{1}$, the decrease in $\mathrm{pH}, \sigma_{f}$ and $[\mathrm{Ca}]$ occurs 12 times later for N04 than for N02, with a flow rate 9 times lower. At high inlet acid concentration $C_{0}^{2}$, we also observe some discrepancies, in particular for N03 and N08, which are submitted to the same experimental conditions, the decrease in the considered measurements occurring 4 times later for N03. Additionally, while the flow rate applied to 

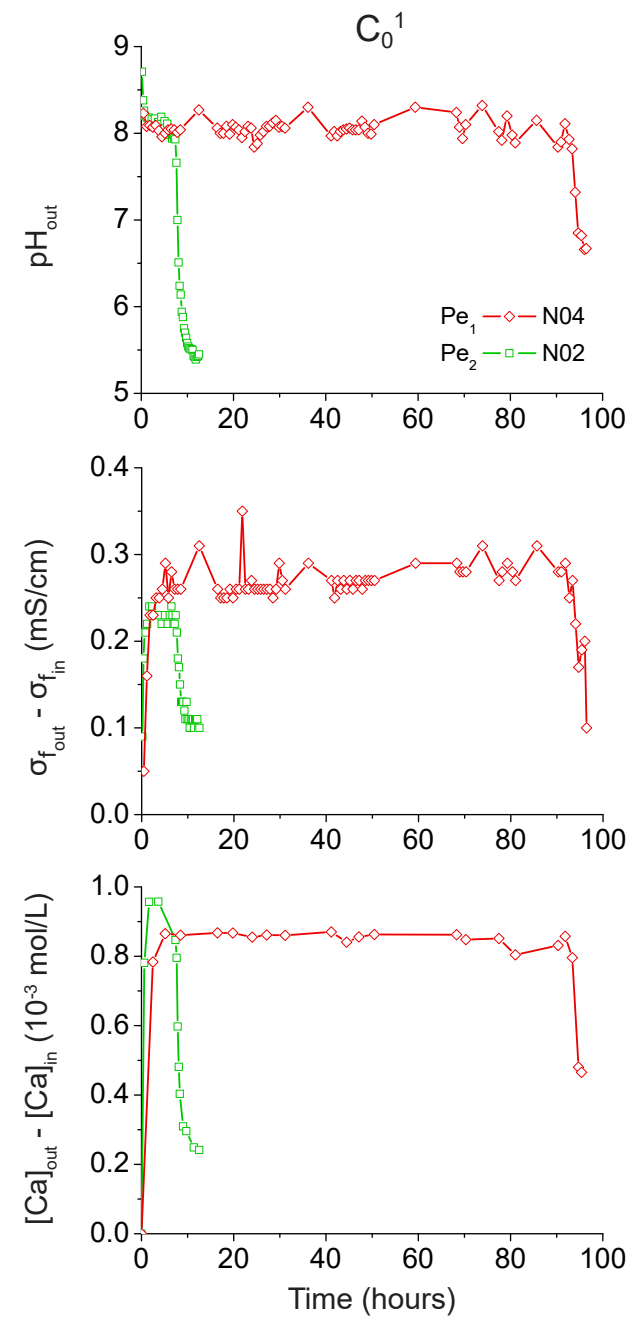

N05 is higher than N08, the experimental parameters decrease slower in the former case than in the latter. All these results show that the differences in time for a same acid concentration are not linear.


Figure 3: Evolution of the $\mathrm{pH}$ at the outlet of the experiments ( $\mathrm{pH}_{\text {out }}$, first row), the differences in fluid conductivity ( $\sigma_{f}$, second row) and calcite concentration ([Ca], third row) between the outlet and inlet of the percolating experiments with inlet acid concentration $C_{0}^{1}$ (first column, empty dots) and $C_{0}^{2}$ (second column, filled dots), and Peclet numbers $P e_{1}$ (diamonds), $P e_{2}$ (squares) and $P e_{3}$ (circles). 
Although the shapes of the curves are similar, the $\mathrm{pH}, \sigma_{f}$ and $[\mathrm{Ca}]$ values observed on the plateau of each experiment are different and depend on the inlet acid concentration. The outlet $\mathrm{pH}$ is one unit higher for $C_{0}^{1}$ than $C_{0}^{2}$, while the outlet $\sigma_{f}$ and $[\mathrm{Ca}]$ are lower when the inlet acid concentration is lower. We observe that the outlet $[\mathrm{Ca}]$ is almost 10 times higher for the experiments performed with $C_{0}^{2}$ than $C_{0}^{1}$, where $C_{0}^{2}$ is 10 times higher than $C_{0}^{1}$.

Figure 4 shows the evolution of permeability over time that is calculated from the monitored differential pressure with the permeability equation provided in Electronic Supplement. When $\mathrm{pH}, \sigma_{f}$ and [Ca] decrease, we observe an increase in permeability. In some cases, a punctual decrease in permeability is observed during the experiments, which is probably due to particles dragging and local pores clogging in the sample, as observed in other studies (Noiriel et al., 2005; Luquot et al., 2014a; Garing et al., 2015). Two permeability trends are shown: (i) an almost constant low permeability until it suddenly increases and reaches the maximum value that is measurable by the sensors for N09 and N04; (ii) an abrupt increase in permeability followed by a moderate increase for the other samples. More specifically, N02 and N08 do not show a permeability increase as large as the other samples since their final relative permeability $k^{*}$ is lower than 100 , whereas it reaches almost 1000 for the other samples. 


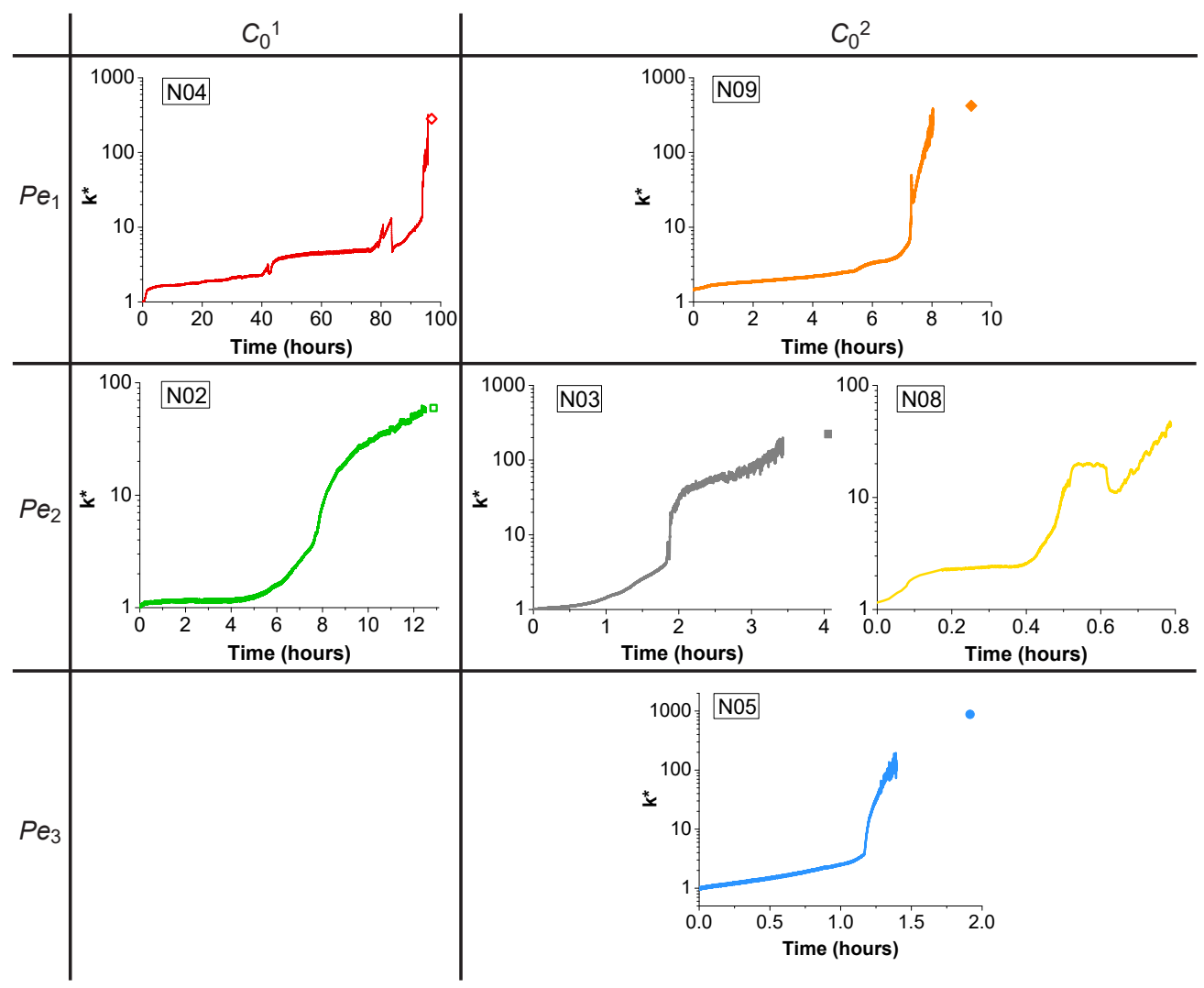

Figure 4: Evolution of the normalized rock permeability $k^{*}[-]$ during the percolating experiments, where $k^{*}$ is defined as the permeability of the sample along the experiment normalized by its initial permeability. The dot represents the final value of permeability. Graphics are displayed according to experimental conditions: two inlet acid concentrations in columns $\left(C_{0}^{1}\right.$ and $\left.C_{0}^{2}\right)$ and three Peclet numbers in rows $\left(P e_{1}, P e_{2}\right.$ and $\left.P e_{3}\right)$.

\subsection{Petrophysical and hydraulic results from laboratory measurements}

\subsubsection{Effective parameters}

Table 2 shows the petrophysical and hydraulic properties obtained from laboratory measurements before and after the percolation experiments. We observe (i) no significant changes for the porosity, (ii) sharp increases for the permeability and the $\mathrm{P}$ and $\mathrm{S}$ waves velocities and (iii) decreases for the structural properties (i.e., formation factor $F_{L}$, cementation index $m_{L}$ and 
209

tortuosity $\tau_{e}$ ).

\begin{tabular}{|c|c|c|c|c|c|c|c|c|c|}
\hline \multirow{7}{*}{. } & Samples & $\phi_{L}$ & \multicolumn{2}{|c|}{$k_{L}$} & $F_{L}$ & $m_{L}$ & $\tau_{e}$ & $V p$ & $V s$ \\
\hline & N02 & 27.9 & \multicolumn{2}{|c|}{3.70} & 54.1 & 3.13 & 3.89 & 2.40 & 1.31 \\
\hline & N03 & 38.0 & \multicolumn{2}{|c|}{11.19} & 8.3 & 2.19 & 1.78 & 2.36 & 1.18 \\
\hline & N04 & 25.6 & \multicolumn{2}{|c|}{1.06} & 50.0 & 2.87 & 3.58 & 3.27 & 1.78 \\
\hline & N05 & 37.8 & \multicolumn{2}{|c|}{9.07} & 7.8 & 2.12 & 1.72 & 2.35 & 1.25 \\
\hline & N08 & 27.7 & \multicolumn{2}{|c|}{8.14} & 26.6 & 2.63 & 2.77 & 2.33 & 1.30 \\
\hline & N09 & 27.2 & \multicolumn{2}{|c|}{0.71} & 24.2 & 2.45 & 2.57 & 2.97 & 1.64 \\
\hline \multirow{7}{*}{$\underset{\Xi}{\overparen{\Xi}}$} & Samples & $\phi_{L}$ & $\phi_{c h}$ & $k_{L}$ & $F_{L}$ & $m_{L}$ & $\tau_{e}$ & $V p$ & $V s$ \\
\hline & N02 & 27.9 & 28.1 & 220 & 17.3 & 2.23 & 2.20 & 3.77 & 1.77 \\
\hline & N03 & 35.7 & 38.3 & 2500 & 5.2 & 1.60 & 1.36 & 2.76 & 1.65 \\
\hline & N04 & 25.8 & 25.9 & 300 & 15.6 & 2.03 & 2.01 & 3.94 & 2.15 \\
\hline & N05 & 36.5 & 38.2 & 8000 & 5.2 & 1.63 & 1.38 & 2.80 & 1.85 \\
\hline & N08 & 29.7 & 28.8 & 230 & 11.7 & 2.03 & 2.03 & 3.74 & 2.01 \\
\hline & N09 & 27.2 & 27.4 & 300 & 15.0 & 2.08 & 2.08 & 3.89 & 1.83 \\
\hline
\end{tabular}

Table 2: Petrophysical properties from laboratory measurements of samples at initial and final states, i.e., before and after the percolation experiments with porosity $\phi_{L}[\%]$, permeability $k_{L}[\mathrm{mD}]$, formation factor $F_{L}[-]$, cementation index $m_{L}[-]$, electrical tortuosity $\tau_{e}[-], \mathrm{P}$ and $\mathrm{S}$ wave velocities $V p$ and $V s[\mathrm{~km} / \mathrm{s}]$. The index $L$ stands for Laboratory measurements and $\phi_{c h}$ is the final porosity calculated from Equation (A.2) with [Ca] measurements

In addition to these behaviors, two groups emerge from the results presented in Table 2. (i) N03 and N05 (gray rows in Table 2) have the highest porosity and permeability, and the lowest electrical factors, at both initial and final states. (ii) The other samples (N02, N04, N08 and N09) show an opposite behavior with N02 displaying the highest electrical factors. In terms of permeability, N05 is the sample displaying the highest increase with $k_{L}$ after experiment almost 1000 times higher than before the experiment, while N02 and N08 permeability only increases around 60 and 30 times, respectively. Concerning $F_{L}$ and $\tau_{e}$, N02 and N04 display the highest decrease compared to N08 and N09. P waves velocities of N02 and N08 increase the 
most compared to N04 and N09, which have the highest $V p$ before and after experiments. Nevertheless, after experiment, N02, N04, N08 and N09 reach similar values. In an opposite way, N03 and N05 keep small values of $V p$ with the lowest increase.

\subsubsection{Pore diameter distribution}

Figure 5 shows the pore diameters distributions that are obtained by centrifugation with very different distributions depending on the drainage direction. When samples are drained in the same direction as the injected flow during experiments, the water stuck into the samples is discharged by the same sample face than during experiments. The results suggest that there are more small pores and less large pores after the dissolution experiment (Figure 5b) than before (Figure 5a). On the contrary, when samples are drained in opposite flow direction, the inlet of samples during experiments is situated at the outlet of the centrifuge. The associated distribution (Figure 5c) is different from that obtained in the main direction, and more similar to the initial one. This difference indicates an anisotropy in the pore size distribution inside the core samples because the water drainage seems easier in the opposite direction of the flow applied during the experiments. This suggests that larger pores are present at the sample inlet. Moreover, after dissolution, a larger amount of small pores present at the sample outlet can prevent the water drainage in this direction, as observed by Luquot et al. (2014b) and Garing et al. (2015). This suggests local particles clogging with a decrease of the throat diameter and an increase of the suction pressure that is needed to drain.

Figure $5 \mathrm{c}$ being the most representative of the pore diameters distribu- 


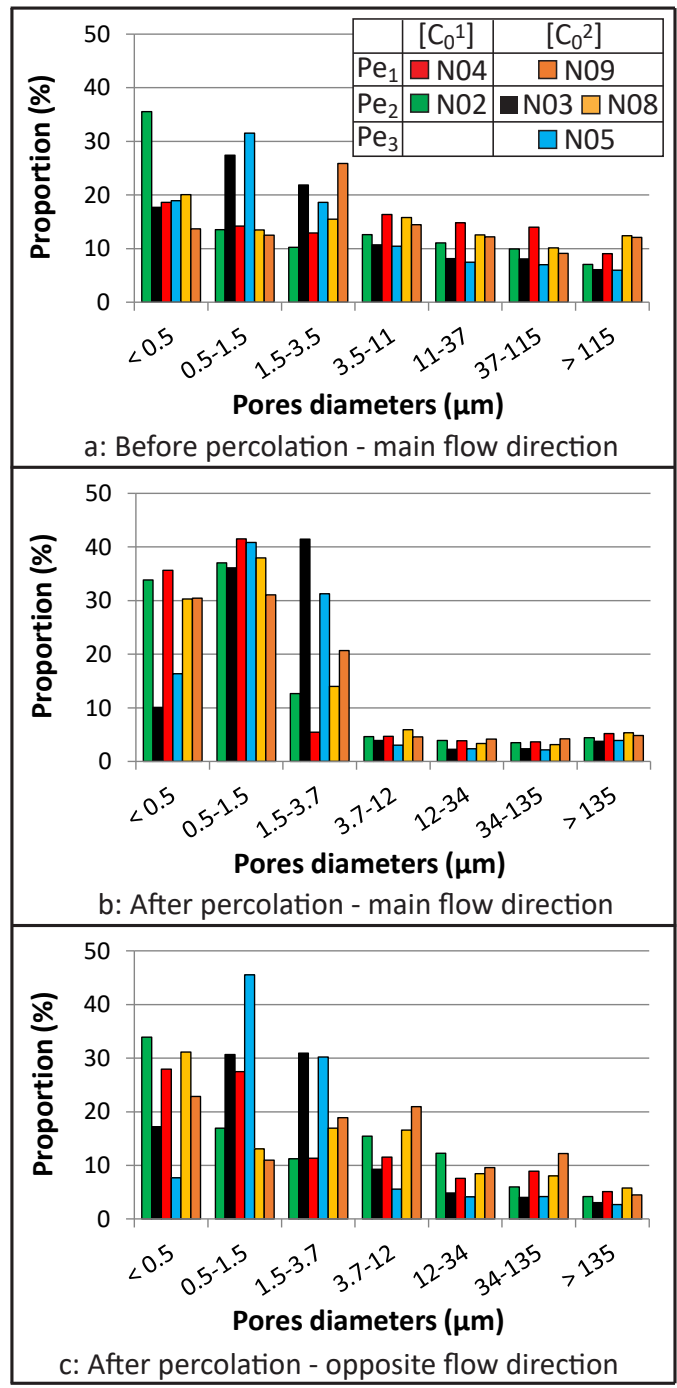

Figure 5: Pore diameters distribution for each sample from centrifugation.

tions, we use these results for comparing the pore diameter distributions before (Figure 5a) and after (Figure 5c) percolation. We observe that the quantity of micropores (pores smaller than $10 \mu \mathrm{m}$ ) increases and that of large pores tends to decrease after percolation. In addition, some groups emerge from these data: (i) N03 and N05 display the largest amount of micropores 
before and after experiment with a moderate increase of $10 \%$ and a larger increase for diameters between 0.5 and $3.5 \mu \mathrm{m}$; (ii) N02 and N09 present the smallest increase in micropores proportion that is around 6\%; (iii) N04 and N08 largely increase their quantity of micropores with increases of $16 \%$ and $13 \%$, respectively. The samples from the group described earlier (Section 3.2.1, samples N02, N04, N08 and N09) do not show the same evolution. N02 has a similar trend before and after percolation, while N09 displays significant increases and decreases in every diameter class. In N04 sample, the proportion of pores with diameters under $1.5 \mu \mathrm{m}$ largely increases, while in N08 the proportion of the smallest pores, under $0.5 \mu \mathrm{m}$, increases.

\subsection{Results from tomographic images and pore network extraction}

\subsubsection{Effective parameters}

Table 3 shows the petrophysical and hydrodynamic properties obtained from the 3D X-ray microtomography images, before and after experiments. We observe that the porosity and permeability increase with the percolation experiments, the formation factor $F_{X R}$ and the ratio of pore surface over pore volume $\frac{S}{V}$ decrease, while no significant changes are observed for the cementation index $m_{X R}$, tortuosity $\tau_{h}$ and percolating volume $P V$.

Samples show some disparities in petrophysical data with, again, some groups emerging. N03 and N05 present the highest increase in permeability, becoming the most permeable samples after experiments, whereas N08 and N09 display the lowest increase. The $\frac{S}{V}$ ratio also increases much more for N03 and N05 than for N08 and N09, which remain almost constant. It should be also noticed that, after experiments, all the samples have similar $\frac{S}{V}$ ratios around $100 \mathrm{~mm}^{-1}$, excepted for N08 with $85 \mathrm{~mm}^{-1}$. Concerning 


\begin{tabular}{|c|c|c|c|c|c|c|c|c|c|}
\hline \multirow{7}{*}{ 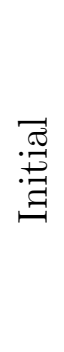 } & Samples & $\phi_{X R}$ & $k_{X R}$ & $k_{P}$ & $F_{X R}$ & $m_{X R}$ & $\tau_{h}$ & $\frac{S}{V}$ & $P V$ \\
\hline & N02 & 7.6 & 36.5 & 4.29 & 40.3 & 1.44 & 1.76 & 107.9 & 92.1 \\
\hline & N03 & 8.3 & 12.6 & 0.21 & 36.2 & 1.44 & 1.74 & 113.0 & 84.7 \\
\hline & N04 & 8.6 & 569 & 94.0 & 15.8 & 1.13 & 1.16 & 80.5 & 96.0 \\
\hline & N05 & 7.9 & 25.5 & 2.14 & 34.1 & 1.39 & 1.64 & 111.4 & 86.1 \\
\hline & N08 & 9.7 & 505 & 367.4 & 28.8 & 1.44 & 1.67 & 87.6 & 95.9 \\
\hline & N09 & 9.1 & 65.2 & 21.1 & 31.3 & 1.43 & 1.68 & 103.0 & 94.9 \\
\hline \multirow{7}{*}{ 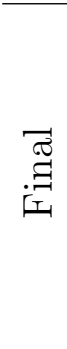 } & Samples & $\phi_{X R}$ & $k_{X R}$ & $k_{P}$ & $F_{X R}$ & $m_{X R}$ & $\tau_{h}$ & $\frac{S}{V}$ & $P V$ \\
\hline & N02 & 8.6 & 1466 & 585.6 & 33.1 & 1.43 & 1.68 & 100.7 & 93.1 \\
\hline & N03 & 8.9 & 8059 & 1983 & 24.7 & 1.33 & 1.48 & 103.5 & 86.4 \\
\hline & N04 & 10.0 & 2674 & 1064 & 25.1 & 1.40 & 1.58 & 97.1 & 94.6 \\
\hline & N05 & 8.8 & 11304 & 3781 & 26.6 & 1.35 & 1.53 & 100.9 & 89.1 \\
\hline & N08 & 10.4 & 1497 & 1056 & 25.5 & 1.43 & 1.63 & 85.0 & 95.7 \\
\hline & N09 & 10.0 & 1291 & 756.7 & 27.3 & 1.44 & 1.65 & 98.9 & 94.8 \\
\hline
\end{tabular}

Table 3: Petrophysical properties from imaging calculations of samples at initial and final states, i.e., before and after the percolation experiments with porosity $\phi_{X R}[\%]$, permeability $k_{X R}$ and $k_{P}[\mathrm{mD}]$, formation factor $F_{X R}[-]$, cementation index $m_{X R}[-]$, tortuosity $\tau_{h}[-]$, ratio of pore surface over pore volume $\frac{S}{V}\left[\mathrm{~mm}^{-1}\right]$ and proportion of percolating volume $P V$ [\%]. $X R$ and $P$ stand for 3D X-ray images and PNM (Pore Network Model) calculations, respectively.

the electrical properties, we can note that N03, and to a lesser extent $\mathrm{N} 05$, present the strongest decrease in $m_{X R}$ and $\tau_{h}$. Before as after experiments, N02 is always the sample with the highest values of $F_{X R}$ and $\tau_{h}$.

\subsubsection{Resulting main flow paths}

Micro-tomography images acquisition and pore network extraction allow to see the main flow paths in each sample after the dissolution experiments, as displayed in Figure 6. For all the core samples, we can observe that preferential flow paths are clearly visible across the entire cores. These main channels were not initially present in the samples, except for sample N08 (Figure 1), and were formed during the percolation experiment and dissolution process. The shape of the main flow path is similar for N02, N04 and 
N09, and is characterized by a relatively straight conduit along sticky pores with few ramifications. N03 and N05 have more ramifications and several inlet points. N05 presents two conduits with only one percolating the entire sample. As mentioned before, N08 is a special case because its percolation follows a pre-existing whole.

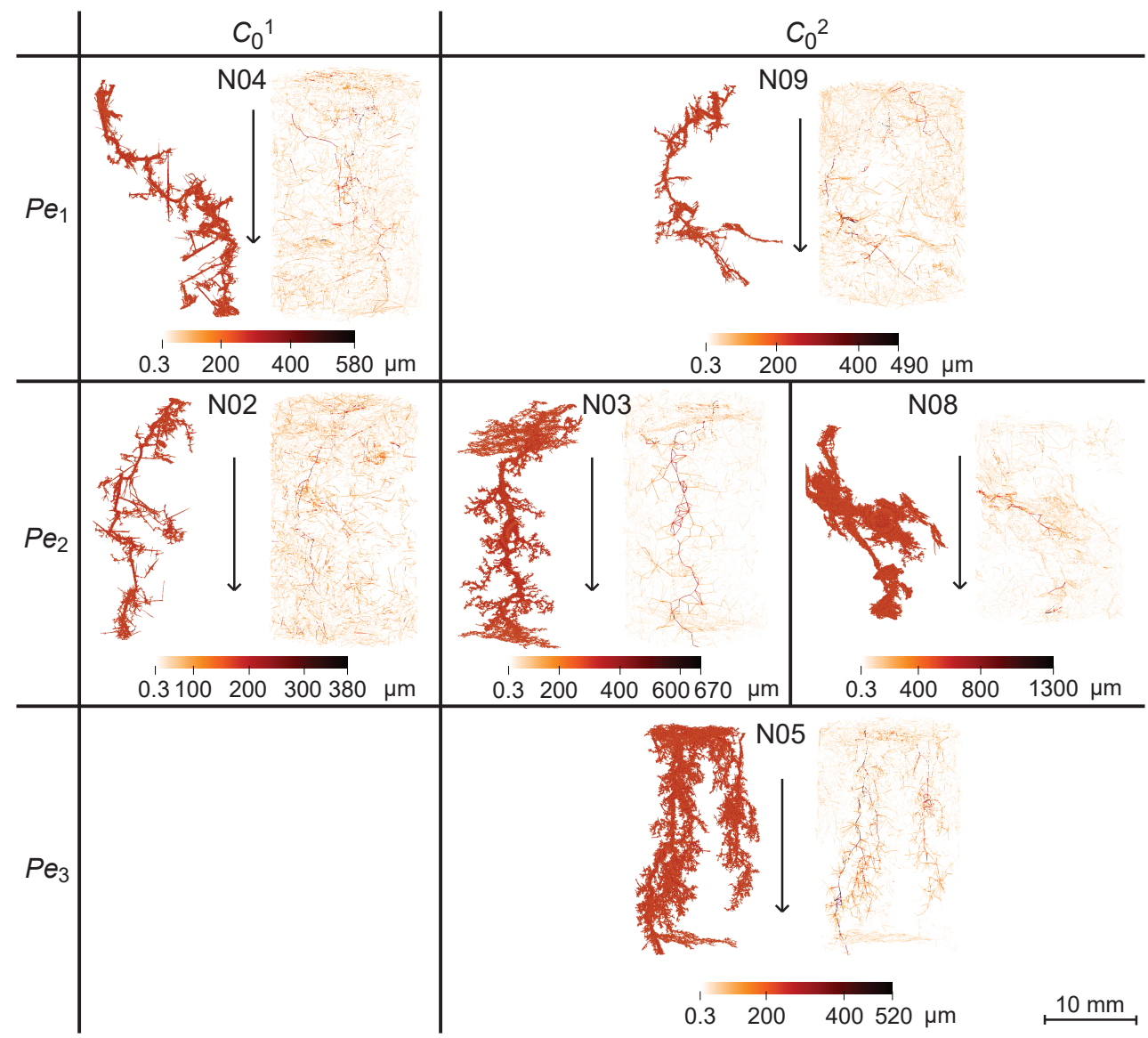

Figure 6: Main path conduits of each sample depending on the experimental conditions: two inlet acid concentrations $C_{0}^{1}$ and $C_{0}^{2}$ in columns and three Peclet numbers $P e_{1}, P e_{2}$ and $\mathrm{Pe}_{3}$ in rows. For each sample, image from tomography acquisition is displayed on the left and image from PNM on the right. The arrow indicates the injected flow direction. The color scales represent the throat diameters in $\mu \mathrm{m}$ extracted from PNM calculations. The scale in the bottom right applies to all samples. 


\subsubsection{Pore size distribution and orientation}

Equivalent pore size distribution is extracted from the 3-D images measuring the chord length probability distribution and is displayed in Figure 7. One of the main advantages of this measure is to statistically get information concerning the pore orientation. We observe here that the pore size increases with the experiments, the proportion of small pores (below $24 \mu \mathrm{m}$ ) decreasing and the proportion of large pores (over $100 \mu \mathrm{m}$ ) increasing. N03 and N05 have changed the most, dividing by two their quantity of small pores and doubling their quantity of large pores. Samples also present an evolution in pore shape. Initially, N02 and N05 display spherical pores with no preferential orientation. With the dissolution, the pore size of N02 increases uniformly in the three space directions and remains spherical, whereas the dissolution enlarges the pores of N05 along the flow direction (Z). Before experiments, N03, N08 and N09 mostly present large pores transverse to the flow direction (XY). After dissolution, large pores of N03 and N09 become longer along the flow direction (Z), while they keep their initial shape in N08. The dissolution does not seem to affect the pore shape of N08, as it follows the initial conduit. N04 has another behaviour with large pores initially extended in $\mathrm{Z}$ direction, which are spherical after the experiment due to the dissolution that enlarges its pores into the transverse flow direction. 


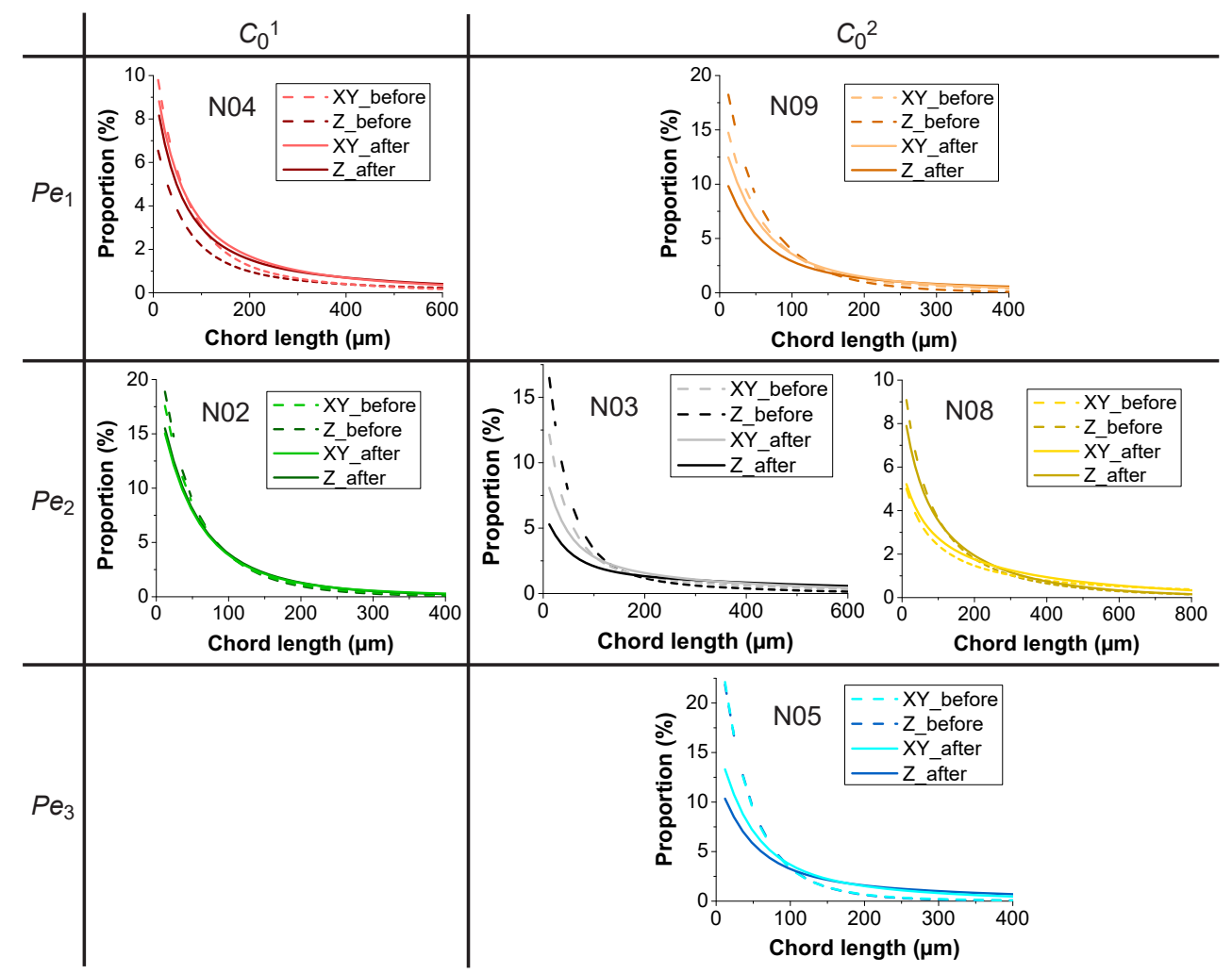

Figure 7: Chord lengths distribution for each sample before (dotted lines) and after (solid lines) percolation. Lines with light colors are for average lengths along $\mathrm{X}$ and $\mathrm{Y}$ axes, while lines with dark colors are for chord lengths along $\mathrm{Z}$ axis.

\section{Discussion}

From the petrophysical results presented in Section 3 and obtained before and after the dissolution experiments, we can divide all the samples into two main groups: Group1 with samples N03 and N05, and Group2 with N02, N04, N08 and N09. In this section, we discuss how these two groups are impacted by the dissolution process and reversely how their specific properties control the reaction localization and rate. 


\subsection{Impact of dissolution on the measured properties}

From the results presented in Section 3, the percolation experiments are responsible for a slight increase in the (chemical) porosity $\left(\phi_{c h}>\phi_{L}\right.$ (initial)), and the macroscopic one evaluated by X-ray microtomography images $\left(\phi_{X R}\right)$ for all the core samples. This is also shown in Figure 8 where the increases in $\phi_{c h}$ range from 0.08 to $0.35 \%$. Even if the increase in porosity seems weak, usual correlated parameters are strongly affected. Velocities of $\mathrm{P}$ and $\mathrm{S}$ waves increase for all the samples (Table 2). This is due to the creation of preferential paths inside the rock (Figure 6) through which the $\mathrm{P}$ and $\mathrm{S}$ waves propagate easily after dissolution. Consistently with their high porosity and their amount of ramifications, samples in Group1 still have the smallest $V p$ after dissolution (Mavko et al., 2009).
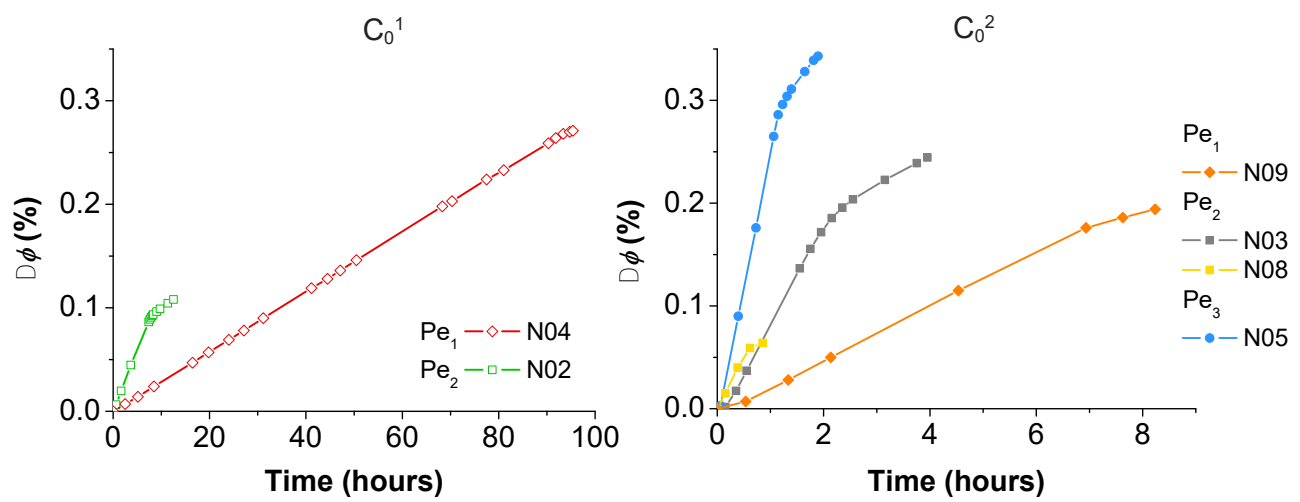

Figure 8: Evolution of rock porosity $\left(\phi_{c h}\right)$ evaluated from Ca ions concentrations analyzed in the outlet fluid during percolating experiments and using Equation (A.2). $\Delta \phi$ is defined as the porosity measured at time $t$ minus the initial porosity $\Delta \phi=\phi(t)-\phi_{i}$.

Usually, the acid passing inside carbonate rocks dissolves the matrix and enlarges the pores (Tutolo et al., 2020). However, after dissolution, the centrifugation results indicate that the quantity of macropores decreases while 
the quantity of micropores increases (Figure 5). This unexpected pore diameters change is due to the particularities in the experimental protocol and in the range of investigation of pore diameters. Indeed, one can expect that the amount of macropores increases during acid fluid percolation (as observed in the XRMT images and in Tutolo et al. (2020)) and especially at the sample inlet, where the acid solution first interacts with the rock. Nevertheless, with the centrifugation method, the amount of large pores is under-estimated because the large pores located at the sample inlet are emptied before the first saturated sample weight measurement and consequently their volume is not taken into account. This under-evaluation can explain the low final laboratory porosity value $\phi_{L}$ compared to the one obtained by mass balance calculation $\phi_{c h}$, as the first one is calculated from the saturated sample weight. Concerning the small pores, the range of investigation of the centrifuge is too small to quantify the amount of pores with diameters under $0.5 \mu \mathrm{m}$. However, the large increase in the smallest measured range indicates that this pore section (smaller than $0.5 \mu \mathrm{m}$ ) increases after the dissolution process as observed by Tutolo et al. (2020), who demonstrated that pores with diameters from $600 \mathrm{~nm}$ to $5 \mu \mathrm{m}$ enlarge preferentially with dissolution process. Even though, the proportion of micropores inside the samples is correlated with their effective porosity. Samples from Group1 have the highest porosity and proportion of micropores with $88 \%$ after dissolution, consistently with Eberli et al. (2003)'s observations.

This phenomenon does not appear in the pore size distribution from chord length calculations (Figure 7). In this case, as expected, the dissolution experiments result in decreasing the amount of small pores and increasing 
the amount of large pores. In Group1, the evolution of pore size distribution is the most significant with an important decrease in the amount of micropores. This implies an important decrease in the ratio $\frac{S}{V}$ (Table 3), where the lowest value of $\frac{S}{V}$ is observed before and after experiments for N08, because of its very low amount of small pores that is observed with all the calculation methods that we used.

We have seen that the dissolution process strongly affects the pore size diameter distribution, as well as others petrophysical parameters. These changes have consequences on other critical properties such as the rock permeability. We already know that small quantity of small pores is usually linked with high permeability (Weger et al., 2009), but some parameters interfere with this correlation. Before rock dissolution, N08 has one of the highest permeability (Tables 2 and 3) correlated with the lowest proportion of small pores (Figures 5 and 7). However, after dissolution, samples from Group1 (N03 and N05) become the most permeable while displaying the highest proportion of small pores. This high proportion of small pores does not prevent a low tortuosity in these samples, usually associated with high permeability. However, it implies a relatively low percolating volume $P V$, around $87 \%$ for Group1 compared to $95 \%$ for Group2. Low tortuosity is also usually directly linked to high connectivity. Before rock dissolution, when preferential conduit does not exist yet, a lot of large pores along the main flow direction are responsible for high tortuosity and low connectivity (Leger and Luquot, 2021). However, dissolution creates preferential paths along the main flow direction, enlarging the pores into this direction and connecting some isolated pores. Therefore, after rock dissolution, a large amount of 
large pores parallel to the main flow direction becomes an indicator of high permeability, low tortuosity and high connectivity.

\subsection{Relationship between rock structure and dissolution process}

Even if the samples groups laid before dissolution mostly remain after it, some inconsistencies are highlighted in the evolution of N08 parameters. In this section, we discuss the role of the initial rock structure on the dissolution patterns and the evolution of petrophysical parameters. We will focus on samples N03 and N08, which have been submitted to similar experimental conditions (Table 1), while they present different initial structures (Figure 1). N03's initial structure is relatively homogeneous while N08 has a pre-existing heterogeneity corresponding to an altered zone with large porosity. Even though, the initial petrophysical properties of N08 are mostly similar to that of the other samples of Group2 (Tables 2 and 3), except the initial permeabilities calculated from images, $k_{X R}$ and $k_{P}$, which are much higher. This is due to the non-visibility of structures smaller than $12 \mu \mathrm{m}$, implying that the calculation considers that there are no small grains obstructing the altered zone and the fluid easily circulates through it. This initial low quantity of small pores (Figures 5 and 7 ) is responsible for the low ratio $\frac{S}{V}$ compared to other samples.

Even if both samples are dissolved with the same experimental conditions, the dissolution gives different results for N03 and N08. Before discussing these differences, we examine the similarities of chemical parameters. In both experiments, the output $\mathrm{pH}$ reaches the same values, as well as the final fluid conductivity and Ca concentration (Figure 3). Nevertheless, the curve breaks in $\mathrm{pH}, \sigma_{f}$, [Ca] (Figure 3), permeability (Figure 4) and poros- 
ity (Figure 8) occur at different times for N03 and N08. As we already know, during the percolation experiments, main flow paths are created (Figure 6). Several previous studies (Daccord, 1987; Fredd and Fogler, 1998; Golfier et al., 2002; Brosse et al., 2005; Szymczak and Ladd, 2009; Luquot and Gouze, 2009; Vialle et al., 2014; Guarracino et al., 2014; Menke et al., 2016) demonstrated that the formation of these preferential paths ends with a channel breakthrough at the sample outlet, inducing some changes in flow location inside the sample. The time at which the channel breaks through at the sample outlet is usually defined as the percolation time $t_{p}$. Here, the conduit in N08 sample percolates 4 times faster than in N03. However, the dissolution rate, defined as $R=[\mathrm{Ca}] \times Q$, is similar for both experiments and around $5 \times 10^{-8} \mathrm{~mol} / \mathrm{s}$. This value of $R$ is consistent with studies performed in similar conditions on others limestone rocks, where $R$ is the same in Noiriel et al. (2009) and is about $2 \times 10^{-8} \mathrm{~mol} / \mathrm{s}$ in Luquot et al. (2014a) and $8 \times 10^{-7} \mathrm{~mol} / \mathrm{s}$ in Vialle et al. (2014). This implies that the quantity of dissolved calcite needed to percolate in N08 sample is 4 times smaller than in N03 sample.

The porosity and permeability of N08 increase much less than that of N03 (Figures 8 and 4). Also, N08 keeps very similar values for electrical parameters in images calculations $\left(F_{X R}, m_{X R}\right.$ and $\tau_{h}$ in Table 3$)$, whereas the pore shape and the pore size distribution remain very similar before and after the experiment (Figures 5 and 7). The main rock structure of the sample N08 does not seem to be really affected by the dissolution process at least at macroscopic scale. This can be explained by the fact that the acid preferentially dissolves the small grains, which present a potentially higher reactive 
surface area, not visible in tomography images. Moreover, the pre-existing channel inside N08 makes the creation of a fully connected path through this sample relatively easy. It only consists in enlarging the initial path by dissolving these small grains and connecting the rest of the sample between the inlet and outlet. Therefore, a short experimental duration is enough to create a percolating path inside this specific sample. The laboratory tortuosity (Table 2) is thus more affected (decreased) than the one measured from XRMT images (Table 3).

To conclude, the structure plays an important role in chalk dissolution in the case of important heterogeneities, leading to fast percolation, low increase in porosity and permeability, and weak changes in pore shapes and pore size distribution, as it has already been observed in fractured samples (Elkhoury et al., 2013; Garcia-Rios et al., 2017).

\subsection{Impact of the experimental conditions on porosity and permeability changes}

Since the pre-existing structural heterogeneity in N08 is responsible for remarkable differences in petrophysical parameters evolution and chemical experimental behavior, it will not be discussed in the following part where Group2 now indicates samples N02, N04 and N09. Focusing on the impact of the experimental conditions on the dissolution and its consequences, we observe that the percolation time $t_{p}$ defined in Section 4.2 is different for each sample (Figures 3, 4 and 8), which are subjected to different experimental conditions (Table 1). The conduit in N04 percolates the latest due to the combination of the lowest flow rate and the lowest inlet acid concentration applied; N02 and N09 conduits percolate at similar faster times around 7.5 hours; and N03 and N05 conduits are created the fastest in 
a
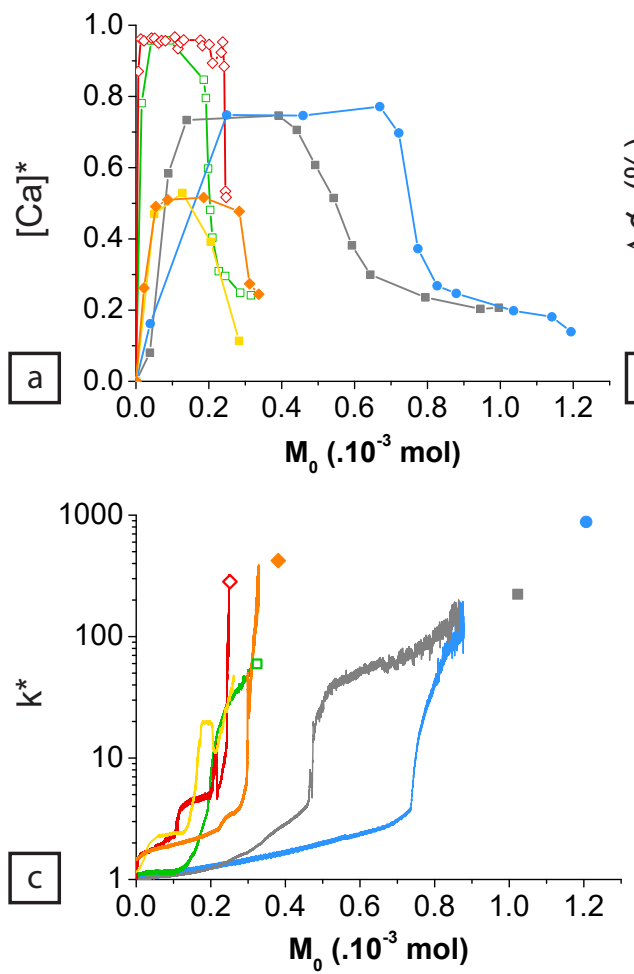

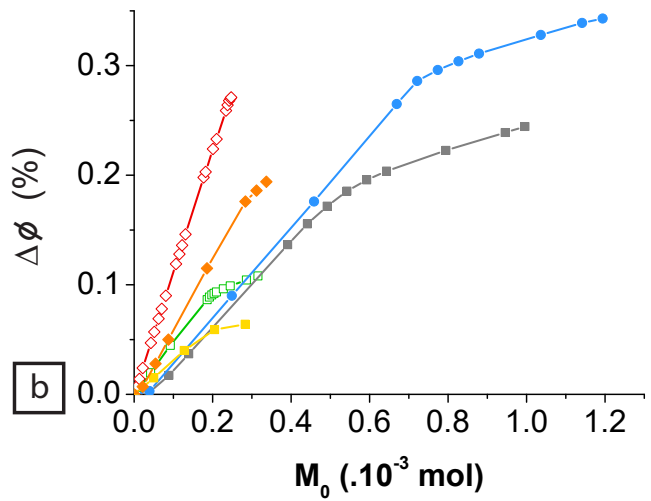

$M_{0}\left(.10^{-3} \mathrm{~mol}\right)$

Figure 9: Evolution of chemical and hydrodynamic fluid parameters during percolating experiments depending on the acid quantity injected over time $M_{0}$ (mol). (a) [Ca] ${ }^{*}$ is the calcite concentration changes between the outlet and inlet normalized by the injected acid concentration, (b) $\Delta \phi$ is the porosity evaluated from Ca concentration changes between the outlet and inlet, (c) $\mathrm{k}^{*}$ is the permeability changes normalized by the initial permeability.

1.9 and 1.2 hours, respectively. In order to understand these differences, Figure 9 displays the relative calcite concentration $[\mathrm{Ca}]^{*}$, the changes in porosity $\Delta \phi$ and the relative permeability $\mathrm{k}^{*}$ according to the quantity of acid injected in the sample over the experiment $M_{0}$ (in mol).

These results lead to the following conclusions: (i) the creation of a percolating conduit in Group2 (N02, N04 and N09) needs a lower quantity of injected acid than Group1 (N03 and N05); (ii) the dissolution behavior depends on the inlet acid concentration; (iii) the quantity of injected acid $M_{0}$ 
until the conduit percolation is not linear with the flow rate applied. More precisely, for $C_{0}^{1}$, the conduit percolation of N02 occurs for a lower $M_{0}$ than the N04 one, while a higher flow rate is applied to N02 sample. On the contrary, for $C_{0}^{2}$, the lower the flow rate is, the lower $M_{0}$ is when conduits percolate. Additionally, Figure 9b displays lower slopes in porosity changes $\Delta \phi$ for Group1 than Group2, which can be explained by the multiple ramifications along samples N03 and N05 being most likely created before the breakthrough (Figure 6). This dissolution shape, closed to homogeneous dissolution, is known to promote more continuous porosity and permeability increases instead of a strong break slope at the percolation time (Luquot and Gouze, 2009). Concerning N04 and N09 from Group2 that display the highest slopes, they are associated with low flow rates that induce transport regimes dominated by diffusion $\left(P e_{1} \ll 1\right)$. This transport type results in an important front dissolution from the sample inlet (Golfier et al., 2002), implying a slow and progressive conduit creation in a tubular way with final reactive surfaces much smaller than before percolation and large paths already created at time $t_{p}$. This induces a sudden high increase in permeability, as it can be observed in Figure 9c. We also observe that N04 has a slope twice higher than N09 in $\Delta \phi$, as well as a twice higher value of calcite concentration $[\mathrm{Ca}]^{*}$. This difference is mostly due to the acid concentration injected since a lower $C_{0}$ injected in N04 with similar flow rates implies that the acid consumption is less important and results in reactions more localized with smaller surfaces. For comparison, N02 and Group1 (N03 and N05) are dissolved with higher flow rates associated with higher Peclet numbers $\left(\mathrm{Pe}_{2}\right.$ and $\left.P_{3}\right)$ and a more advective acid transport inside the samples. This is 
responsible for the permeability changes $k^{*}$ patterns, where the permeability still increases after the conduit percolation. In this case, the reaction surface inside these samples being larger (more ramifications), the reaction takes place at more locations than in samples with conduits more tubular (Noiriel et al., 2009), increasing the quantity of rock dissolved.

These results are completed with the good correlation that we observe between our data and the well-known power-law relating permeability and porosity $k=\phi^{n}$ (Kozeny, 1927; Carman, 1937) shown in Figure 10. Exponent $n$ values found from the power law stand between 140 (N05) and 338 (N02), which are much higher than those found in the literature, with for instance $n=3$ for granular packings in constant structure formation in Kozeny (1927) and Carman (1937), or $1<n<2.5$ for channels formation in limestone dissolution (Beckingham, 2017; Sabo and Beckingham, 2021). Higher values are found in the literature when studying limestone dissolution with $5<$ $n<11$ (Menke et al., 2015, 2016), $n=5$ (Poonoosamy et al., 2020), $n=13$ (Noiriel et al., 2004), $4<n<24$ (Garing et al., 2015), $10<n<20$ (Bernabé et al., 2003) and $n=75$ (Noiriel et al., 2005). This is mostly due to the important heterogeneities created during the reaction in limestone (Brace, 1980; Lucia, 1995; Vialle et al., 2013; Menke et al., 2016) and the relationship between the permeability and the pores roughness (Noiriel et al., 2004) and connectivity (Gueguen and Dienes, 1989), the latter being not homogeneous inside a karstified sample (Vialle et al., 2013). Here, the values are higher than the values found in the literature, except for some fracture samples (Noiriel et al., 2005), which highlights the strong heterogeneities created in our rock samples. In addition, we can mentioned that the lowest $n$ value is 


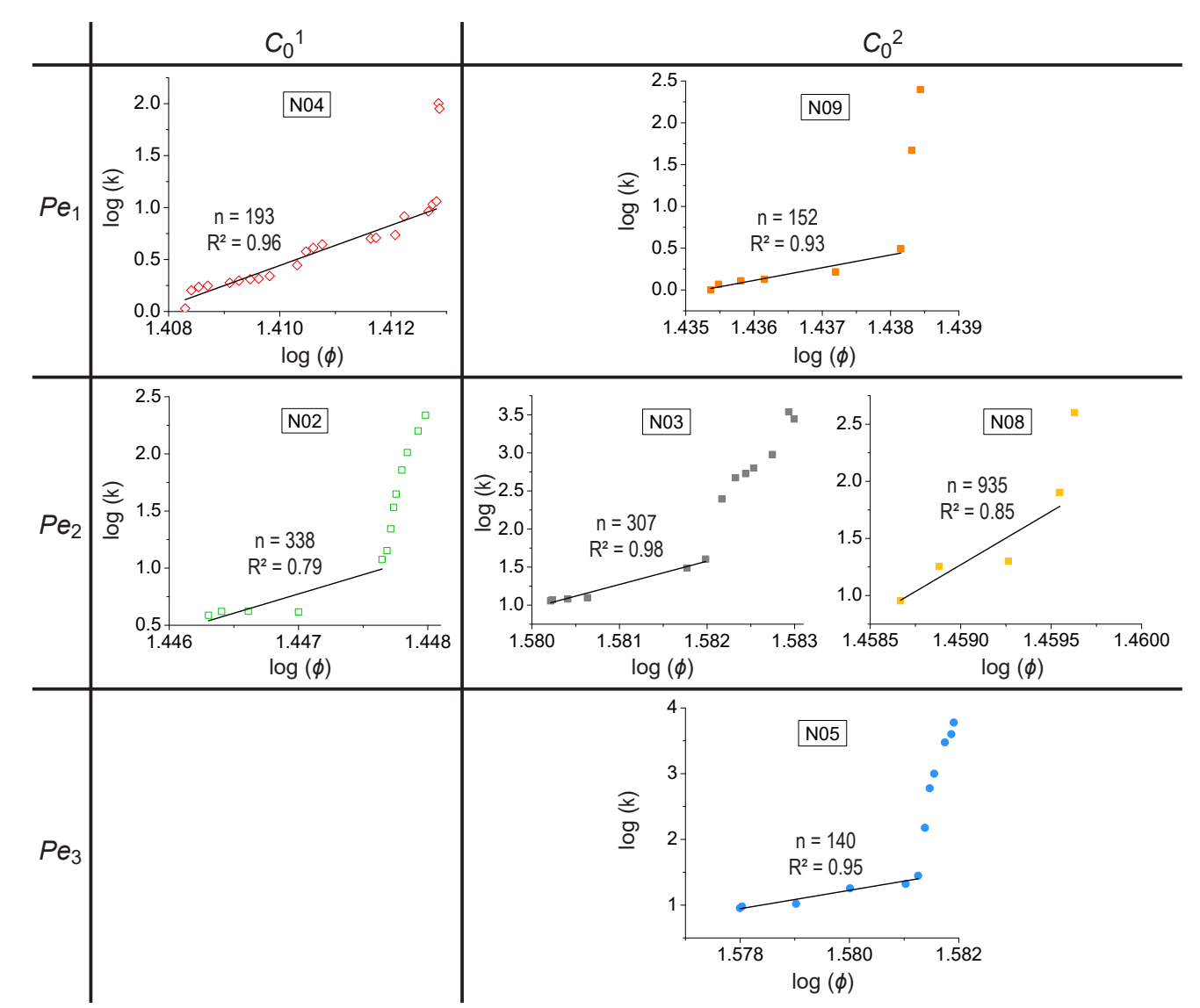
the largest one is obtained for N08 characterized by a strong heterogeneity. Attempt to improve the porosity-permeability relationship with a critical porosity as done in Luquot and Gouze (2009) was not successful in this work and requires deeper analysis.

Figure 10: Permeability over porosity for all the samples. $n$ is the power exponent from

Figure 10: Permeability over porosi
the Kozeny-Carman law $k=\phi^{n}$.

obtained for sample N05 with the most ramified dissolution process, whereas

4.4. Linking the dissolution rate and percolation time with the Damköhler number

Damköhler number definition 
Considering that the experiments are conducted under diffusion-dominant transport regimes ( $P e \leq 1$ except for N05) and focusing on Damköhler number expressions that do not require to define the reaction rate $K$, we use expressions (1) and (2) developed by Knapp (1989) and Menke et al. (2015), respectively,

$$
\begin{gathered}
D a=\frac{\alpha S_{r} r L^{2}}{C_{\mathrm{eq}} d} \\
D a=\frac{\pi r l}{n_{\mathrm{Ca}} d} .
\end{gathered}
$$

In expression (1), $\alpha$ is the stoechiometric coefficient of element $i$ in the mineral $\left(\alpha=1\right.$ for $\mathrm{Ca}$ in calcite $\left.\mathrm{CaCO}_{3}\right) ; S_{r}$ is the reactive specific surface area $\left[\mathrm{m}^{-1}\right]$ set to either the ratio $\frac{S}{V}$ that is provided in Table 3 or $\frac{S}{V_{\mathrm{Ca}}}$ with $V_{\mathrm{Ca}}$ the calcite volume in each sample; $r$ is the kinetic rate for a flat and pure sample (calcite) with no transport limitation $\left[\mathrm{mol} / \mathrm{m}^{2} / \mathrm{s}\right] ; L$ is the characteristic length of the system set to the length of the pores $l$ as for diffusion-dominant regimes (Menke et al., 2015) and $C_{\text {eq }}$ is the equilibrium concentration of element $i\left(\mathrm{Ca}^{2+}\right.$, here $)[\mathrm{mol} / \mathrm{L}]$. The kinetic rates $r$ are extrapolated from values found in Pokrovsky et al. (2009). For a temperature of $25^{\circ} \mathrm{C}$ and an equivalent $\mathrm{CO}_{2}$ partial pressure of 2 and 7 atm, corresponding to the $C_{0}^{1}$ and $C_{0}^{2}$ acid concentrations respectively, the equivalent kinetic rates are $3 \times 10^{-5} \mathrm{~mol} / \mathrm{m}^{2} / \mathrm{s}$ and $5 \times 10^{-5} \mathrm{~mol} / \mathrm{m}^{2} / \mathrm{s}$, respectively. Note also that the parameter $S_{r}$, usually considered as a fitting parameter (Noiriel and Daval, 2017), is not well defined because (i) it is underestimated when using the ratio $\frac{S}{V}$ obtained by imagery calculations that ignore small pores (smaller than the pixel size) and (ii) it requires to determine the saturation index $\Omega$ that is set to 0 here when using the ratio $\frac{S}{V_{\mathrm{Ca}}}$ (Luquot and Gouze, 2009). 
In expression (2), $n_{\mathrm{Ca}}$ is the number of moles of calcite per volume unit $\left[\mathrm{mol} / \mathrm{m}^{3}\right]$ defined as $n_{\mathrm{Ca}}=\frac{\rho_{\text {calcite }}(1-\phi)}{M_{\text {calcite }}}$ and equals to $1.7 \times 10^{4} \mathrm{~mol} / \mathrm{m}^{3}$ for the most porous samples (Group1) and $2 \times 10^{4} \mathrm{~mol} / \mathrm{m}^{3}$ for the least porous samples (Group2).

Figure 11 show the Damköhler numbers $D a_{\frac{S}{V}}$ and $D a_{\frac{S}{V_{\text {Ca }}}}$ obtained with expression (1) by setting $S_{r}$ to $\frac{S}{V}$ and $\frac{S}{V_{\mathrm{Ca}}}$, respectively, and $D a_{M}$ obtained with expression (2). $D a_{\frac{S}{V}}$ presents the lowest values and $D a_{\frac{S}{V_{\text {Ca }}}}$ the highest because the values of $\frac{S}{V}$ are much smaller than that of $\frac{S}{V_{\mathrm{Ca}}}$. $D a_{M}$ values are similar to $D a_{\frac{S}{V_{\mathrm{Ca}}}}$ with slightly lower values as well as less variability between the samples. $D a_{\frac{S}{V}}$ seems not to be the most representative calculation because of the debatable choice of $S_{r}$ as $\frac{S}{V}$ (Noiriel and Daval, 2017). However, it is difficult to determinate which is the correct one, each one having is own definition, approximation and hypothesis. In all the cases, Da numbers are lower than 1 , meaning that the reaction rate is low compared to the transport rate standing for an uniform dissolution according to Golfier et al. (2002). However, Figure 6 displays conduits in each sample, meaning that the dissolution is not uniform, as observed by Menke et al. (2016) who found similar results and conclude to a channelisation dissolution regime. To create this dissolution pattern, the flow rate is relatively high inside the sample, and the rock heterogeneities allow a preferential conduit to form where the reactant is focused. During our dissolution experiments, the flow and Pe number are much lower than in Menke et al. (2016) experiments and even in these conditions the initial structural rock properties seem to control the localization and shape of the reaction.

Dissolution rate and percolation time analysis 


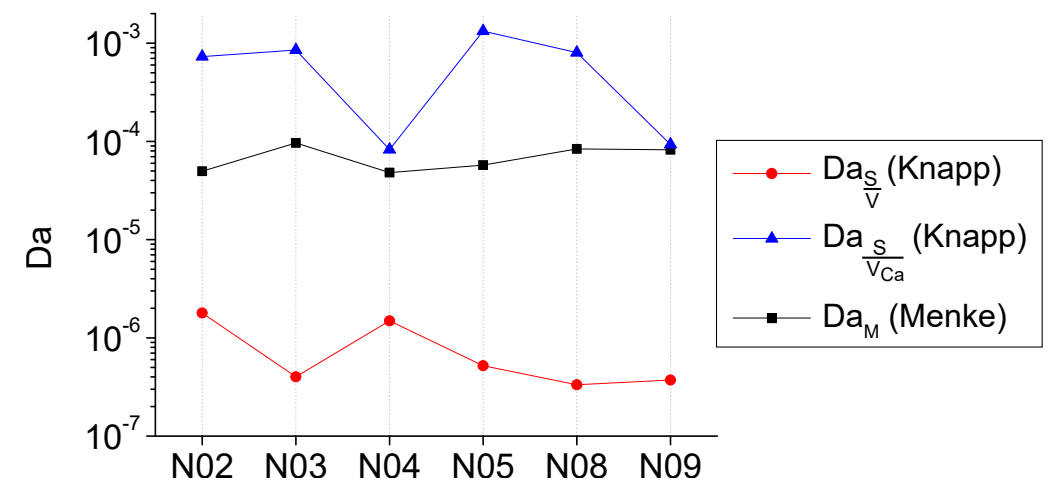

Figure 11: Damkohler numbers from three different calculations. $D a_{\frac{S}{Y}}$ (red circles) and $D a_{\frac{S}{V_{\mathrm{Ca}}}}$ (blue triangles) (Knapp, 1989) display results from Equation (1) with $S_{r}$ either set to $\frac{S}{V}$ or $\frac{S}{V_{\mathrm{Ca}}}$. $D a_{M}$ (black squares) (Menke et al., 2015) presents results from Equation (2).

Figure 12 and Table 4 show the evolution of the dissolution rate $R$ and percolation time $t_{p}$ depending on the experimental conditions (Pe and Da numbers).

The increase in flow rate $\left(\frac{Q_{N 03}}{Q_{N 09}}=9\right.$ and $\left.\frac{Q_{N 05}}{Q_{N 09}}=22\right)$ induces an increase in Pe numbers for samples N09, N03 and N05. The corresponding Da numbers are quite constant for those calculated with the Menke equation $\left(D a_{M}\right)$ while $D a_{\frac{S}{V_{\mathrm{Ca}}}}$ increases with Pe increase. The dissolution rate increases linearly with the Pe number (Figure 12 and Table 4) while the percolation time $t_{p}$ (Figure 12) decreases with Pe increase and Da decrease (for $D a_{V_{\overparen{ }}}$ ). However, the total quantity of dissolved calcite until breakthrough $\left([\mathrm{Ca}]_{d}\right)$ and the quantity of dissolved calcite per quantity of injected acid $\left([\mathrm{Ca}]^{\#}\right)$ increase with the Pe increase (Table 4). These changes are higher between N09 and N03 compared to N03 and N05 because of the differences in flow rates injected into the samples. This is also related to the difference in conduits shape after experiments (Figure 6), N09 having a tubular conduit while N03 


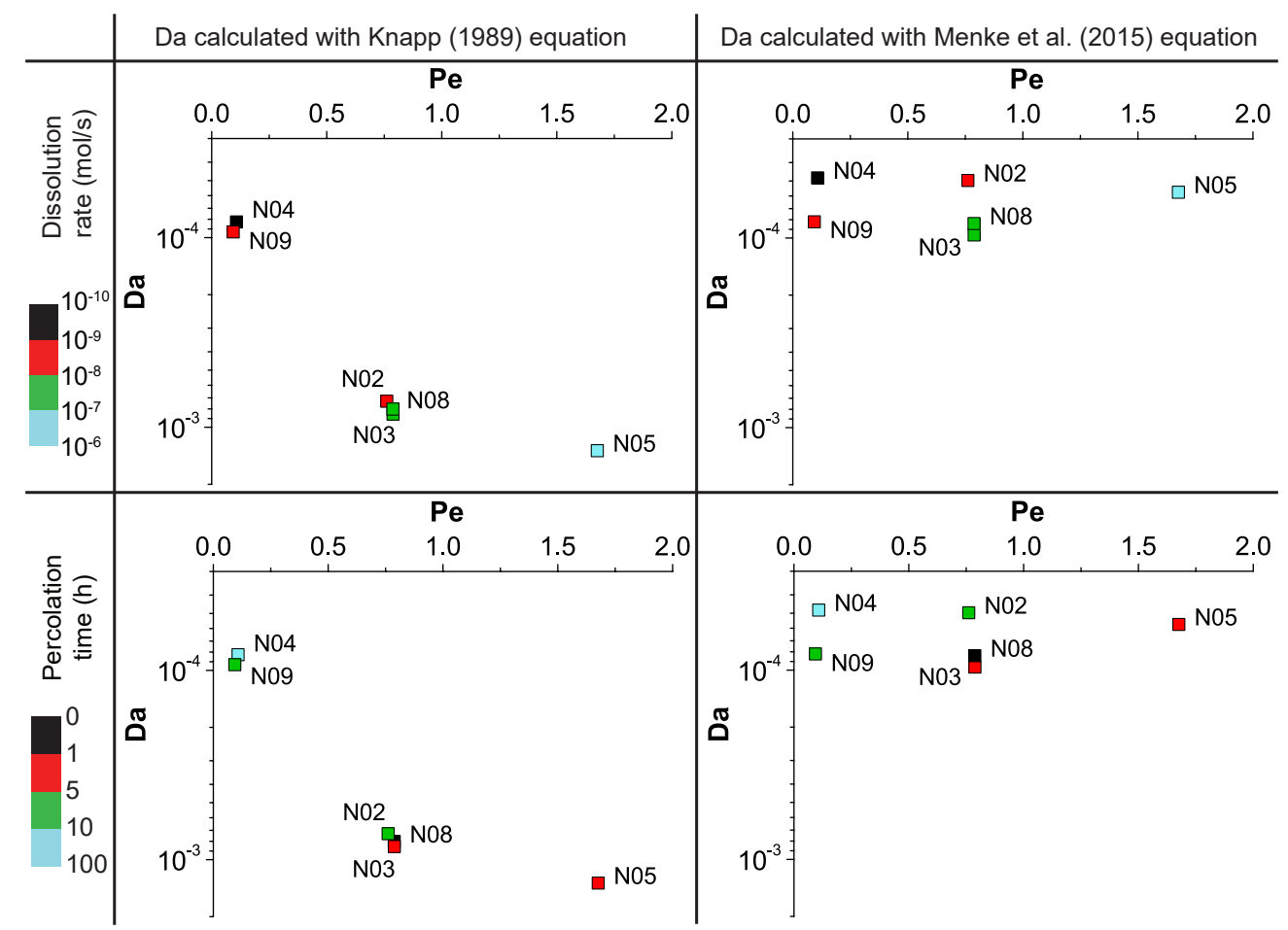

Figure 12: Samples with their values of dissolution rate $R$ (on the top) and percolation time $t_{p}$ (on the bottom) plotted depending on Pe and Da numbers. On the left, samples are displayed for Da calculated with Equation (1), while on the right, they are displayed for the calculations with Equation (2).

and N05 have ramified conduits.

For the considered injected acid concentration, a change of Pe conditions, from $\mathrm{Pe}_{1}$ to $\mathrm{Pe}_{2}$, induces an increase by one order of magnitude in the dissolution rate (Figure 12). On the contrary, the percolation time depends on the acid concentration as its decrease is higher for a high acid concentration. The dissolution rates for N02 and N09 are quite similar as well as the dissolution shape, characterized by tubular conduit (Figure 6). Consequently, the Pe and Da diagram does not help us to predict the dissolution shape. Nevertheless, these observations allow seeing that a 10 times increase in acid 


\begin{tabular}{cccccccc} 
Samples & $\mathrm{Pe}$ & $D a_{M}$ & $D a_{\frac{S}{V_{C a}}}$ & $R$ & $t_{p}$ & {$[\mathrm{Ca}]_{d}$} & {$[\mathrm{Ca}]^{\#}$} \\
\hline N02 & 0.76 & $4.97 \mathrm{e}^{-5}$ & $7.30 \mathrm{e}^{-4}$ & $6.69 \mathrm{e}^{-9}$ & 7.77 & $1.87 \mathrm{e}^{-4}$ & 0.96 \\
N03 & 0.79 & $9.64 \mathrm{e}^{-5}$ & $8.52 \mathrm{e}^{-4}$ & $5.17 \mathrm{e}^{-8}$ & 1.88 & $3.49 \mathrm{e}^{-4}$ & 0.74 \\
N04 & 0.11 & $4.81 \mathrm{e}^{-5}$ & $8.26 \mathrm{e}^{-5}$ & $6.89 \mathrm{e}^{-10}$ & 93.92 & $2.33 \mathrm{e}^{-4}$ & 0.96 \\
N05 & 1.68 & $5.73 \mathrm{e}^{-5}$ & $1.33 \mathrm{e}^{-3}$ & $1.31 \mathrm{e}^{-7}$ & 1.17 & $5.48 \mathrm{e}^{-4}$ & 0.75 \\
N08 & 0.79 & $8.38 \mathrm{e}^{-5}$ & $8.02 \mathrm{e}^{-4}$ & $4.49 \mathrm{e}^{-8}$ & 0.45 & $7.27 \mathrm{e}^{-5}$ & 0.49 \\
N09 & 0.09 & $8.21 \mathrm{e}^{-5}$ & $9.33 \mathrm{e}^{-5}$ & $5.86 \mathrm{e}^{-9}$ & 7.27 & $1.53 \mathrm{e}^{-4}$ & 0.52
\end{tabular}

Table 4: Table of experimental properties of each sample. Pe is the average Pe calculated with lowest and largest $l, D a_{M}$ is calculated from Equation (2), Da $\frac{S}{\nabla_{C a}}$ is calculated from Equation (1), $R$ is the dissolution rate $[\mathrm{mol} / \mathrm{s}], t_{p}$ is the percolation time $[\mathrm{h}],[\mathrm{Ca}]_{d}$ is the total quantity of dissolved calcite until conduit percolation [mol] and [Ca] ${ }^{\#}$ is the quantity of dissolved calcite per quantity of injected acid $[\mathrm{mol} / \mathrm{mol}]$.

concentration and a 9 times increase in flow rate have similar consequences on the dissolution. Indeed, these changes lead to similar decreases in percolation times $t_{p}$ and dissolved calcite rates $[\mathrm{Ca}]_{d}$. N02 and N09 conduits both percolate around 7.5 hours with a $[\mathrm{Ca}]_{d}$ around $1.7 \mathrm{e}^{-4} \mathrm{~mol}$, and present similar conduits shape (Figure 6). N09 conduit percolates faster than the N02 one because of the pre-existing micro-cracks inside the sample, which make easier the conduit formation.

To sum up, in the case of Da calculated with Equation (1) $\left(D a_{\frac{S}{V_{C a}}}\right)$, for each injected acid concentration, higher the Pe is, higher the Da is. Therefore, more the transport is advective (Pe increases), more the reaction is dominant compared to the transport (Da increases). However, in the case of Da calculated with Equation (2) $\left(D a_{M}\right)$, the increase in Pe is not associated with changes in Da. Also, as seen earlier, a low Da is logically linked with a low $C_{0}$. For sample N05, the Da is relatively low due to the advective dominated transport. The dissolution rate $R$ follows $P$ and $D a_{\frac{S}{V_{C a}}}$, since it is smaller when both numbers are smaller. However, it is also sharply 
related to the acid concentration, which is much smaller when $C_{0}$ is smaller for similar Pe and Da conditions.

\section{Conclusions}

In this study, we try to identify which parameter most controls the chalk dissolution at pore scale and then the karstification. The flow rate and the initial concentration of acid injected inside the rock seem to be important factors regarding dissolution patterns. Dissolution rates of calcite and percolation time of formed conduits are directly linked to these two parameters. However, the structure seems to also have a decisive impact on dissolution. We previously see that chalk is defined by an important heterogeneous microstructure (Leger and Luquot, 2021), composed of a lot of small components, leading to a high structural heterogeneity. This is responsible for special patterns during dissolution. The local heterogeneities induce a very large exponent in the porosity-permeability power law of Kozeny (1927) and Carman (1937). The dissolution regime inside each sample is dependent on the rock structure, as it has been shown in several previous studies. In samples with high initial porosity (N03 and N05), the surface reaction is larger and the dissolution is more uniform than in samples with lower initial porosity (N02, N04, N08 and N09). Also, rock heterogeneities are responsible for channel formation inside the samples during the dissolution while calculated Peclet and Damköhler numbers define an uniform dissolution, as previously demonstrated in Menke et al. (2016). In this study, we could reproduce dissolution features similar to mini-karst as observed at larger scale in chalk environments. We demonstrate that the localization of the chalk dissolution 
635 is mainly linked to the specific small scale heterogeneities and not directly 636 predictable using dimensionless parameters such as Da and Pe numbers. 


\section{Appendix A. Experimental procedure}

\section{Appendix A.1. Experimental protocol}

The following protocol is applied to each sample. The sample is placed under vacuum condition during a minimum of 12 hours. Then, the sample is saturated at low flow rate with water in equilibrium with the rock. Equilibrium water is then injected at increasing and decreasing flow rates in order to clean the pore phase and remove free particles into the samples. When pressure changes are linear with flow rate changes, the sample is considered cleaned and flow rate is fixed at the wanted one. The initial permeability is deduced at this starting stage from the pressure drop and the given flow rate with the equation provided in Electronic Supplement. Then, the acid solution is injected through the core sample. Experiments are considered over when pressure sensors are out of measurement capabilities. At this point, water at equilibrium with rock is injected in order to calculate a final rock permeability. The flow rate is increased slowly and step by step to avoid to damage the sample. This protocol is applied to experiments that are conducted with various acid concentrations and flow rate. The acid concentrations are all buffered to a $\mathrm{pH}$ of 4 and the flow rates are chosen such as the experiments could be done over reasonable time to observe the effect of acid injection while being low enough to limit grains separations.

\section{Appendix A.2. Experimental output}

For each experiment, the evolution of the sample porosity $\phi(t)$ along time is deduced from the measured concentration of $\mathrm{Ca}$ ions with the following methodology. From the pressure measurements, the permeability $k$ is calculated using Darcy's law (equation provided in Electronic Supplement). 
Concentration of $\mathrm{Ca}$ ions are measured for each outlet sampled fluid that is collected at time $t_{i}(i=1, \ldots, N)$ with $N$ the number of samples. The porosity can be deduced from this concentration $[\mathrm{Ca}]_{t_{i}}$ with the following steps. (i) The calcium carbonate mass $m_{\mathrm{CaCO}_{3 t_{i}}}$ dissolved between times $t_{i-1}$ and $t_{i}$ is calculated from Equation (A.1), which is based on the equivalence between the quantity of Ca measured in the sampled fluid and the quantity of dissolved $\mathrm{CaCO}_{3}$ from the rock, in mol, and expressed as

$$
m_{\mathrm{CaCO}_{3 t_{i}}}=\frac{\left([\mathrm{Ca}]_{t_{i}}-[\mathrm{Ca}]_{t_{0}}\right)\left(t_{i}-t_{i-1}\right) Q}{M_{\mathrm{Ca}}} M_{\mathrm{CaCO}_{3}}
$$

with $[\mathrm{Ca}]_{t_{i}}$ the calcium concentration at the sampling time $t_{i}\left[\mathrm{~g} / \mathrm{m}^{3}\right],[\mathrm{Ca}]_{t_{0}}$ the initial calcium concentration $\left[\mathrm{g} / \mathrm{m}^{3}\right], t_{i}-t_{i-1}$ the time spent since the last sampling $[\mathrm{s}], Q$ the flow rate $\left[\mathrm{m}^{3} / \mathrm{s}\right]$, and $M_{\mathrm{Ca}}$ and $M_{\mathrm{CaCO}_{3}}$ the molecular masses of calcium and calcium carbonate, respectively $[\mathrm{g} / \mathrm{mol}]$. (ii) The volume of dissolved calcium carbonate in the rock sample between each sampling step, $V_{\mathrm{CaCO}_{3 t_{i}}}$, is obtained by dividing $m_{\mathrm{CaCO}_{3 t_{i}}}$ by the density of calcium carbonate that is set to $2.7 \times 10^{6} \mathrm{~g} / \mathrm{m}^{3}$. (iii) The porosity $\phi_{t_{i}}$ is deduced from the following expression

$$
\phi_{t_{i}}=\phi_{t_{i-1}}+\frac{V_{\mathrm{CaCO}_{3 t_{i}}}}{V_{\text {ech }}}
$$

with $V_{e c h}$ the sample volume $\left[\mathrm{m}^{3}\right]$. The initialization of the previous equation for $t=0$ is made by using the initial laboratory porosity $\phi_{L}$. 


\section{Electronic Supplement: Procedure and algorithm for OpenPNM software}

Procedure for pore network extraction

The 3D images obtained from X-ray tomography calculations are used to extract the pore network of the samples with the open-source software OpenPNM that is described below (Gostick et al., 2016). The permeability $k$ of the resulting extracted pore networks is calculated with Darcy's law as

$$
k=\frac{Q L \mu}{A \Delta P},
$$

where $Q$ is the flow rate $\left[\mathrm{m}^{3} / \mathrm{s}\right], L$ the sample length $[\mathrm{m}], \mu$ the dynamic viscosity of water [Pa.s] set to 0.001 Pa.s, $A$ the surface of fluid injection $\left[\mathrm{m}^{2}\right]$ and $\Delta P$ the differential pressure between the inlet and outlet of the sample $[\mathrm{Pa}]$. The flow rate resulting from a chosen gradient pressure that is applied to the sample is determined by Stokes equation.

OpenPNM tool is working on Scipy Stack from Anaconda navigator, which is a Python console. The method used here is the watershed segmentation compiled with the SNOW algorithm, previously used and explained by Gostick (2017). The extraction process needs segmented images in 8 bits format with two distinct labels: 0 for pore voxels and 255 for rock voxels. Our images from chalk samples being too large for OpenPNM, a binning of 2 is applied on each image by dividing the number of voxels by 2 in the three spatial directions. Additionally, we only consider the connected porosity by removing the isolated pores. Once the segmented image is imported, an Euclidian distance map of the void space and a Gaussian filter with the parameter sigma set to 0.4 are applied. It results in an image with one peak 
at each pore center, based on a maximum radius of 5 pixels. Then, some filters are implemented to clean false generated peaks and obtain a map of pore centers. The next step consists in applying the watershed segmentation to identify regions where pores are located. The SNOW algorithm is then used to extract the pore network from the image, generating the network composed of pores and throats. Then, a resolution label of 0.01 is applied to the network in order to identify which pores are located on sample surfaces. The network can be exported into different file formats for visualization and treatments. To operate permeability computation on the network, a cleaning step is necessary, consisting in removing isolated pores. In our case, the image has been cleaned previously, so this step is not changing the network.

Algorithm for pore network extraction and permeability computation Algorithm used on segmented images with OpenPNM

\section{print('Read libraries')}

import os

import imageio

import scipy as sp

import numpy as np

import openpnm as op

import porespy as ps

import scipy.ndimage as spim

import matplotlib.pyplot as plt

from porespy.filters import find_peaks, trim_saddle_points, trim_nearby_peaks from porespy.tools import randomize_colors

from skimage.segmentation import watershed 
ps.visualization.set_mpl_style()

np.set_printoptions(precision $=4$ )

np.random.seed(10)

\section{print('Read image')}

path $=$ 'path to image folder'

file_format $=$ '.tif'

file_name = 'filename'

file $=$ file_name + file_format

fetch_file = os.path.join(path, file)

im = imageio.mimread(fetch_file, memtest='image_size')

$\mathrm{im}=\mathrm{np} \cdot \operatorname{array}(\mathrm{im}, \mathrm{dtype}=\mathrm{bool})$

print('Gaussian filter')

sigma $=0.4$

$\mathrm{dt}=$ spim.distance_transform_edt(input $=\mathrm{im})$

$\mathrm{dt}=$ spim.gaussian_filter $($ input $=\mathrm{dt}, \operatorname{sigma}=$ sigma $)$

print('Identify peaks')

peaks $=$ find_peaks $\left(\mathrm{dt}=\mathrm{dt}, \mathrm{r} \_\max =5\right)$

print('Initial number of peaks: ', spim.label(peaks)[1])

peaks $=$ trim_saddle_points $($ peaks $=$ peaks, $\mathrm{dt}=\mathrm{dt})$

print('Peaks after trimming saddle points: ', spim.label(peaks)[1])

peaks $=$ trim_nearby_peaks $($ peaks $=$ peaks, $\mathrm{dt}=\mathrm{dt})$

peaks, $\mathrm{N}=$ spim.label(peaks)

print('Peaks after trimming nearby peaks: ', N)

print('Regionalyze step')

regions $=$ watershed $($ image $=-\mathrm{dt}$, markers $=$ peaks, mask $=\mathrm{dt}>0)$ 


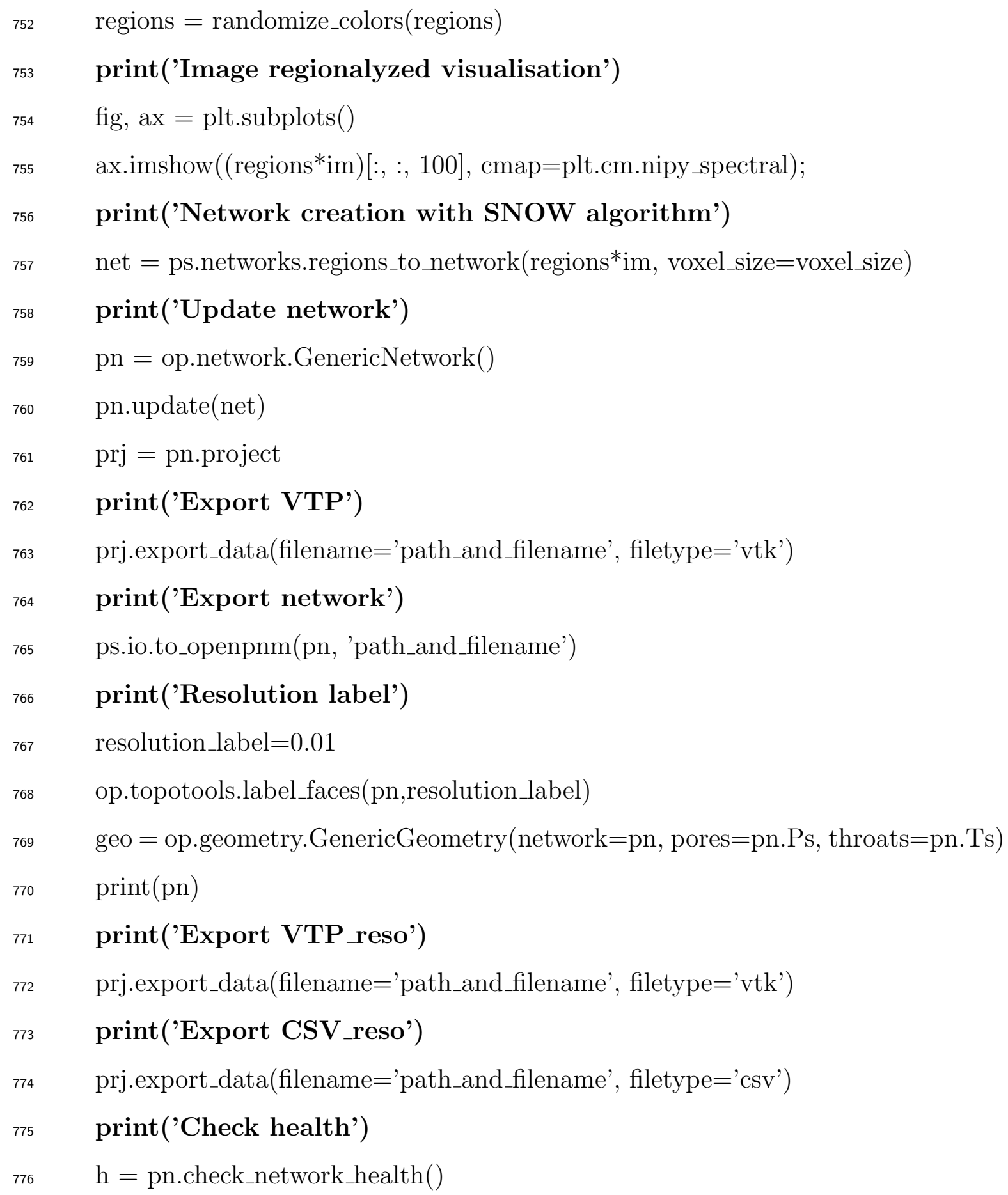




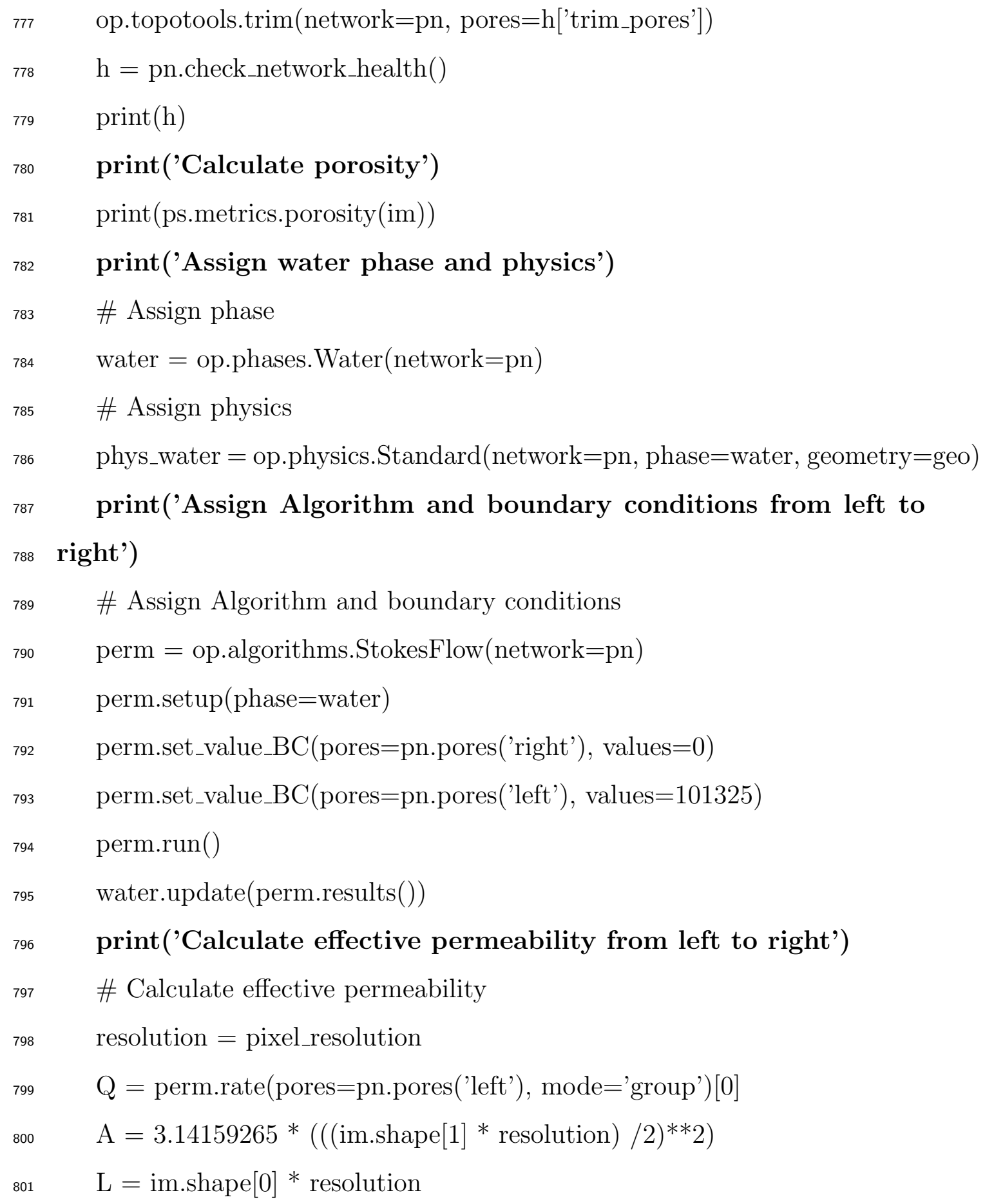




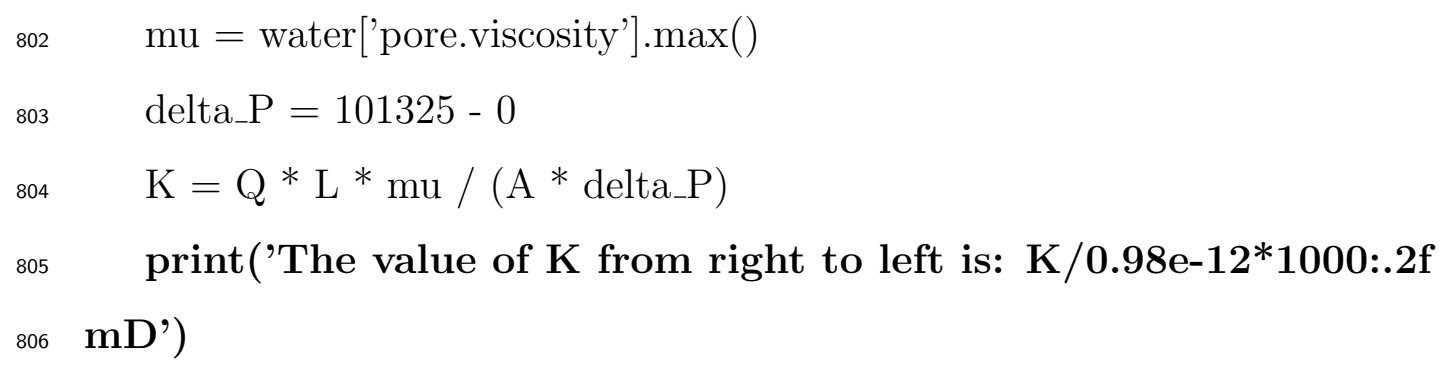


807

808

809

810

811

812

813

814

815

\section{Acknowledgements}

This research was funded by the research projects EC2CO (INSU-CNRS) StarTrek and JPI-Water UrbanWat from WaterWorks2017, and by a CIFRE PhD fellowship provided by Voxaya and ANRT. We acknowledge the MRI platform member of the national infrastructure France-BioImaging supported by the French National Research Agency (ANR-10-INBS-04, "Investments for the future"), the labex CEMEB (ANR-10-LABX-0004) and NUMEV (ANR-10-LABX-0020). We also acknowledge the petrophysics plateform members of Geosciences Montpellier Laboratory. 


\section{References}

Beckingham, L.E., 2017. Evaluation of Macroscopic Porosity-Permeability Relationships in Heterogeneous Mineral Dissolution and Precipitation Scenarios. Water Resour. Res. 53, 10217-10230. doi:10.1002/2017WR021306.

Bernabé, Y., Mok, U., Evans, B., 2003. Permeability-porosity Relationships in Rocks Subjected to Various Evolution Processes. Pure appl. geophys. 160, 937-960. doi:10.1007/PL00012574.

Brace, W., 1980. Permeability of crystalline and argillaceous rocks. International Journal of Rock Mechanics and Mining Sciences \& Geomechanics Abstracts 17, 241-251. doi:10.1016/0148-9062(80)90807-4.

Brosse, E., Magnier, C., Vincent, B., 2005. Modelling Fluid-Rock Interaction Induced by the Percolation of CO2 -Enriched Solutions in Core Samples: the Role of Reactive Surface Area. Oil \& Gas Science and Technology Rev. IFP 60, 287-305. doi:10.2516/ogst:2005018.

Carman, P.C., 1937. Fluid Flow through Granular Beds. Trans. Inst. Chem. Eng. 15, 150-166.

Chilingarian, G.V., Mazzullo, S.J., Rieke, H.H., 1992. Carbonate reservoir characterization: a geologic-engineering analysis. Number 30, 44 in Developments in petroleum science, Elsevier, Amsterdam ; New York, NY, USA.

Choquette, P.W., Pray, L.C., 1970. Geologic Nomenclature and Classification of Porosity in Sedimentary Carbonates. Bulletin 54. doi:10.1306/5D25C98B-16C1-11D7-8645000102C1865D. 
Daccord, G., 1987. Chemical dissolution of a porous medium by a reactive fluid. Phys. Rev. Lett. 58, 479-482. doi:10.1103/PhysRevLett.58.479.

Daccord, G., Lenormand, R., Liétard, O., 1993. Chemical dissolution of a porous medium by a reactive fluid-I. Model for the "wormholing" phenomenon. Chemical Engineering Science 48, 169-178. doi:10.1016/00092509(93)80293-Y.

Eberli, G.P., Baechle, G.T., Anselmetti, F.S., Incze, M.L., 2003. Factors controlling elastic properties in carbonate sediments and rocks. The Leading Edge 22, 654-660. doi:10.1190/1.1599691.

Elkhoury, J.E., Ameli, P., Detwiler, R.L., 2013. Dissolution and deformation in fractured carbonates caused by flow of CO2-rich brine under reservoir conditions. International Journal of Greenhouse Gas Control 16, S203S215. doi:10.1016/j.ijggc.2013.02.023.

Fabricius, I.L., 2007. Chalk: composition, diagenesis and physical properties. bgsd 55, 97-128. doi:10.37570/bgsd-2007-55-08.

Ford, D., Williams, P., 2007. Karst Hydrogeology and Geomorphology: Ford/Karst Hydrogeology and Geomorphology. John Wiley \& Sons Ltd,., West Sussex, England. doi:10.1002/9781118684986.

Fredd, C.N., Fogler, H.S., 1998. Influence of transport and reaction on wormhole formation in porous media. AIChE J. 44, 1933-1949. doi:10.1002/aic.690440902.

Garcia-Rios, M., Luquot, L., Soler, J.M., Cama, J., 2015. Influence of the flow rate on dissolution and precipitation features during percolation of 
CO2-rich sulfate solutions through fractured limestone samples. Chemical Geology 414, 95-108. doi:10.1016/j.chemgeo.2015.09.005.

Garcia-Rios, M., Luquot, L., Soler, J.M., Cama, J., 2017. The role of mineral heterogeneity on the hydrogeochemical response of two fractured reservoir rocks in contact with dissolved CO2. Applied Geochemistry 84, 202-217. doi:10.1016/j.apgeochem.2017.06.008.

Garing, C., Gouze, P., Kassab, M., Riva, M., Guadagnini, A., 2015. Anticorrelated Porosity-Permeability Changes During the Dissolution of Carbonate Rocks: Experimental Evidences and Modeling. Transp Porous Med 107, 595-621. doi:10.1007/s11242-015-0456-2.

Gharbi, O., Bijeljic, B., Boek, E., Blunt, M.J., 2013. Changes in Pore Structure and Connectivity Induced by CO2 Injection in Carbonates: A Combined Pore-Scale Approach. Energy Procedia 37, 5367-5378. doi:10.1016/j.egypro.2013.06.455.

Golfier, F., Zarcone, C., Bazin, B., Lenormand, R., Lasseux, D., Quintard, M., 2002. On the ability of a Darcy-scale model to capture wormhole formation during the dissolution of a porous medium. Journal of Fluid Mechanics 457. doi:10.1017/S0022112002007735.

Gostick, J., Aghighi, M., Hinebaugh, J., Tranter, T., Hoeh, M.A., Day, H., Spellacy, B., Sharqawy, M.H., Bazylak, A., Burns, A., Lehnert, W., Putz, A., 2016. OpenPNM: A Pore Network Modeling Package. Comput. Sci. Eng. 18, 60-74. doi:10.1109/MCSE.2016.49. 
Gostick, J.T., 2017. Versatile and efficient pore network extraction method using marker-based watershed segmentation. Phys. Rev. E 96, 023307. doi:10.1103/PhysRevE.96.023307.

Guarracino, L., Rötting, T., Carrera, J., 2014. A fractal model to describe the evolution of multiphase flow properties during mineral dissolution. Advances in Water Resources 67, 78-86. doi:10.1016/j.advwatres.2014.02.011.

Gueguen, Y., Dienes, J., 1989. Transport properties of rocks from statistics and percolation. Math Geol 21, 1-13. doi:10.1007/BF00897237.

Hoefner, M.L., Fogler, H.S., 1988. Pore evolution and channel formation during flow and reaction in porous media. AIChE J. 34, 45-54. doi:10.1002/aic.690340107.

Knapp, R.B., 1989. Spatial and temporal scales of local equilibrium in dynamic fluid-rock systems. Geochimica et Cosmochimica Acta 53, 19551964. doi:10.1016/0016-7037(89)90316-5.

Kozeny, J., 1927. Uber kapillare Leitung des Wassers im Boden. Sitzungberichte der Akadamie der Wissenschaftung in Wien Abteilung Ila , 271301.

Lasaga, A.C., 1984. Chemical kinetics of water-rock interactions. J. Geophys. Res. 89, 4009-4025. doi:10.1029/JB089iB06p04009.

Lebedev, M., Zhang, Y., Sarmadivaleh, M., Barifcani, A., Al-Khdheeawi, E., Iglauer, S., 2017. Carbon geosequestration in limestone: Pore-scale dissolution and geomechanical weakening. International Journal of Greenhouse Gas Control 66, 106-119. doi:10.1016/j.ijggc.2017.09.016. 
Leger, M., Luquot, L., 2021. Importance of Microstructure in Carbonate Rocks: Laboratory and 3D-Imaging Petrophysical Characterization. Applied Sciences 11, 3784. doi:10.3390/app11093784.

Lucia, F., 1983. Petrophysical Parameters Estimated From Visual Descriptions of Carbonate Rocks: A Field Classification of Carbonate Pore Space. Journal of Petroleum Technology 35, 629-637. doi:10.2118/10073-PA.

Lucia, F.J., 1995. Rock-Fabric petrophysical classification of carbonate porespace for reservoir characterization. AAPG Bulletin-American Association of Petroleum Geologists 79, 1275-1300.

Luhmann, A.J., Kong, X.Z., Tutolo, B.M., Garapati, N., Bagley, B.C., Saar, M.O., Seyfried, W.E., 2014. Experimental dissolution of dolomite by CO2-charged brine at $100^{\circ} \mathrm{C}$ and 150 bar: Evolution of porosity, permeability, and reactive surface area. Chemical Geology 380, 145-160. doi:10.1016/j.chemgeo.2014.05.001.

Luquot, L., Gouze, P., 2009. Experimental determination of porosity and permeability changes induced by injection of $\mathrm{CO} 2$ into carbonate rocks. Chemical Geology 265, 148-159. doi:10.1016/j.chemgeo.2009.03.028.

Luquot, L., Rodriguez, O., Gouze, P., 2014a. Experimental Characterization of Porosity Structure and Transport Property Changes in Limestone Undergoing Different Dissolution Regimes. Transp Porous Med 101, 507-532. doi:10.1007/s11242-013-0257-4.

Luquot, L., Roetting, T.S., Carrera, J., 2014b. Characterization of flow parameters and evidence of pore clogging during limestone 
dissolution experiments. Water Resources Research 50, 6305-6321. doi:10.1002/2013WR015193.

Lønøy, A., 2006. Making sense of carbonate pore systems. Bulletin 90, 1381-1405. doi:10.1306/03130605104.

Mangane, P.O., Gouze, P., Luquot, L., 2013. Permeability impairment of a limestone reservoir triggered by heterogeneous dissolution and particles migration during CO2-rich injection: dissolution-induced particle migration. Geophys. Res. Lett. 40, 4614-4619. doi:10.1002/grl.50595.

de Marsily, G., 1986. Quantitative hydrogeology; groundwater hydrology for engineers. Academic Press .

Mavko, G., Mukerji, T., Dvorkin, J., 2009. The rock physics handbook: tools for seismic analysis of porous media. 2nd ed ed., Cambridge University Press, Cambridge, UK ; New York. OCLC: ocn268793772.

Menke, H., Andrew, M., Blunt, M., Bijeljic, B., 2016. Reservoir condition imaging of reactive transport in heterogeneous carbonates using fast synchrotron tomography — Effect of initial pore structure and flow conditions. Chemical Geology 428, 15-26. doi:10.1016/j.chemgeo.2016.02.030.

Menke, H., Bijeljic, B., Blunt, M., 2017. Dynamic reservoir-condition microtomography of reactive transport in complex carbonates: Effect of initial pore structure and initial brine $\mathrm{pH}$. Geochimica et Cosmochimica Acta 204, 267-285. doi:10.1016/j.gca.2017.01.053. 
Menke, H.P., Bijeljic, B., Andrew, M.G., Blunt, M.J., 2015. Dynamic ThreeDimensional Pore-Scale Imaging of Reaction in a Carbonate at Reservoir Conditions. Environ. Sci. Technol. 49, 4407-4414. doi:10.1021/es505789f.

Molins, S., Trebotich, D., Yang, L., Ajo-Franklin, J.B., Ligocki, T.J., Shen, C., Steefel, C.I., 2014. Pore-Scale Controls on Calcite Dissolution Rates from Flow-through Laboratory and Numerical Experiments. Environmental Science \& Technology 48, 7453-7460. doi:10.1021/es5013438.

Moore, C.H., Wade, W.J., 2013. Carbonate reservoirs: porosity and diagenesis in a sequence stratigraphic framework. Number 67 in Developments in sedimentology. second edition ed., Elsevier, Amsterdam.

Noiriel, C., Bernard, D., Gouze, P., Thibault, X., 2005. Hydraulic Properties and Microgeometry Evolution Accompanying Limestone Dissolution by Acidic Water. Oil \& Gas Science and Technology 60, 177-192. doi:10.2516/ogst:2005011.

Noiriel, C., Daval, D., 2017. Pore-Scale Geochemical Reactivity Associated with CO2 Storage: New Frontiers at the Fluid-Solid Interface. Acc. Chem. Res. 50, 759-768. doi:10.1021/acs.accounts.7b00019.

Noiriel, C., Gouze, P., Bernard, D., 2004. Investigation of porosity and permeability effects from microstructure changes during limestone dissolution. Geophysical Research Letters 31. doi:10.1029/2004GL021572.

Noiriel, C., Luquot, L., Madé, B., Raimbault, L., Gouze, P., van der Lee, J., 2009. Changes in reactive surface area during limestone dissolution: 
An experimental and modelling study. Chemical Geology 265, 160-170. doi:10.1016/j.chemgeo.2009.01.032.

Pokrovsky, O.S., Golubev, S.V., Schott, J., Castillo, A., 2009. Calcite, dolomite and magnesite dissolution kinetics in aqueous solutions at acid to circumneutral $\mathrm{pH}, 25$ to $150{ }^{\circ} \mathrm{C}$ and 1 to 55 atm pCO2: New constraints on CO2 sequestration in sedimentary basins. Chemical Geology 265, 20-32. doi:10.1016/j.chemgeo.2009.01.013.

Poonoosamy, J., Klinkenberg, M., Deissmann, G., Brandt, F., Bosbach, D., Mäder, U., Kosakowski, G., 2020. Effects of solution supersaturation on barite precipitation in porous media and consequences on permeability: Experiments and modelling. Geochimica et Cosmochimica Acta 270, 4360. doi:10.1016/j.gca.2019.11.018.

Rege, S.D., Fogler, H.S., 1989. Competition among flow, dissolution, and precipitation in porous media. AIChE J. 35, 1177-1185. doi:10.1002/aic.690350713.

Sabo, M.S., Beckingham, L.E., 2021. Porosity-Permeability Evolution During Simultaneous Mineral Dissolution and Precipitation. Water Res 57. doi:10.1029/2020WR029072.

Singurindy, O., Berkowitz, B., 2003. Flow, dissolution, and precipitation in dolomite. Water Resour. Res. 39. doi:10.1029/2002WR001624.

Smith, M.M., Sholokhova, Y., Hao, Y., Carroll, S.A., 2013. Evaporite Caprock Integrity: An Experimental Study of Reactive Mineralogy and 
Pore-Scale Heterogeneity during Brine-CO2 Exposure. Environmental Science \& Technology 47, 262-268. doi:10.1021/es3012723.

Steefel, C.I., Beckingham, L.E., Landrot, G., 2015. Micro-Continuum Approaches for Modeling Pore-Scale Geochemical Processes. Reviews in Mineralogy and Geochemistry 80, 217-246. doi:10.2138/rmg.2015.80.07.

Szymczak, P., Ladd, A.J.C., 2009. Wormhole formation in dissolving fractures. J. Geophys. Res. 114, B06203. doi:10.1029/2008JB006122.

Tutolo, B., Luhmann, A., Kong, X.Z., Bagley, B., Alba-Venero, D., Mitchell, N., Saar, M., Seyfried, Jr., W., 2020. Contributions of visible and invisible pores to reactive transport in dolomite. Geochem. Persp. Let. , 42-46doi:10.7185/geochemlet.2022.

Tutolo, B.M., Luhmann, A.J., Kong, X.Z., Saar, M.O., Seyfried, W.E., 2014. Experimental Observation of Permeability Changes In Dolomite at CO2 Sequestration Conditions. Environ. Sci. Technol. 48, 2445-2452. doi:10.1021/es4036946.

Vialle, S., Contraires, S., Zinzsner, B., Clavaud, J., Mahiouz, K., Zuddas, P., Zamora, M., 2014. Percolation of CO2 -rich fluids in a limestone sample: Evolution of hydraulic, electrical, chemical, and structural properties. J. Geophys. Res. Solid Earth 119, 2828-2847. doi:10.1002/2013JB010656.

Vialle, S., Dvorkin, J., Mavko, G., 2013. Implications of pore microgeometry heterogeneity for the movement and chemical reactivity of $\mathrm{CO} 2$ in carbonates. GEOPHYSICS 78, L69-L86. doi:10.1190/geo2012-0458.1. 
${ }_{1017}$ Weger, R.J., Eberli, G.P., Baechle, G.T., Massaferro, J.L., Sun, Y.F., 2009. 1018 Quantification of pore structure and its effect on sonic velocity and perme1019 ability in carbonates. Bulletin 93, 1297-1317. doi:10.1306/05270909001. 1020 Yang, Y., Hakim, S.S., Bruns, S., Rogowska, M., Boehnert, S., Hammel, 1021 J.U., Stipp, S.L.S., Sørensen, H.O., 2018. Direct Observation of Coupled 1022 Geochemical and Geomechanical Impacts on Chalk Microstructure Evolu1023 tion under Elevated CO2 Pressure. ACS Earth Space Chem. 2, 618-633. 1024 doi:10.1021/acsearthspacechem.8b00013. 